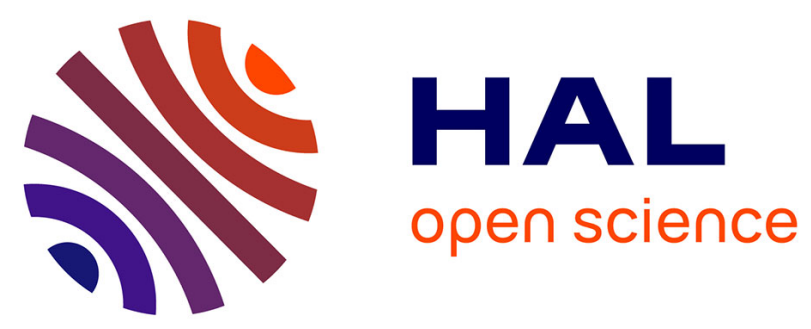

\title{
Gradual College Admission *
}

Guillaume Haeringer, Vincent Iehlé

\section{To cite this version:}

Guillaume Haeringer, Vincent Iehlé. Gradual College Admission *. Journal of Economic Theory, 2021, 198 (105378), 10.1016/j.jet.2021.105378 . halshs-02330435v2

\section{HAL Id: halshs-02330435 \\ https://shs.hal.science/halshs-02330435v2}

Submitted on 10 Mar 2021

HAL is a multi-disciplinary open access archive for the deposit and dissemination of scientific research documents, whether they are published or not. The documents may come from teaching and research institutions in France or abroad, or from public or private research centers.
L'archive ouverte pluridisciplinaire HAL, est destinée au dépôt et à la diffusion de documents scientifiques de niveau recherche, publiés ou non, émanant des établissements d'enseignement et de recherche français ou étrangers, des laboratoires publics ou privés. 


\title{
Gradual College Admission*
}

\author{
Guillaume Haeringer $^{\dagger} \quad$ Vincent Iehlé
}

March 4, 2021

\begin{abstract}
We study multi-period college admission problems where, at each period, a matching is computed and students have the option to either finalize their matches or participate to the next period. Students participating to an additional run of the matching mechanism can submit a new rank order list to the matching clearinghouse. Such gradual matching systems can adequately account for an additional source of heterogeneity among participants, like withdrawals. We identify the conditions under which such systems first ensure that participating to additional runs of the matching mechanism is safe for participants (in the sense that they can secure the spot they obtained at the previous round) and second yield to stable matchings (with a stability concept adapted to this environment). We use our results to evaluate the former French college admission system, where students could finalize their matches at different dates up to two months ahead the end of the admission campaign.
\end{abstract}

JEL classification: C78, D02

Keywords: gradual matching, withdrawal, French college admissions system.

*We thank Laura Doval, Lucien Frys, Victor Hiller, Fuhito Kojima, Juan Sebastián Pereyra, Olivier Tercieux, Xavier Venel, Alex Westkamp, Bumin Yenmez, and seminar and conference participants at Paris School of Economics, Université Paris 1 Panthéon-Sorbonne, Université Paris 2 Panthéon-Assas, CSEF Università Federico II, Ottawa Theory Workshop, EWGE in Glasgow, SING in Odense, SAET in Faro and Pittsburgh-Carnegie Mellon. We are also extremely grateful to three anonymous reviewers and the Associate Editor for their extensive and thoughtful comments and criticisms about our earlier draft. Both authors gratefully acknowledge the support of ANR grant Dynamite-13BSH1-0010.

$\dagger$ Baruch College.

${ }_{\ddagger}^{\ddagger}$ Normandie Université, UNIROUEN, CREAM. 


\section{Introduction}

In the matching literature the standard design for a mechanism consists of first asking participants to submit a rank order list (ROL) over potential matches and then run an algorithm to compute a matching. It is then assumed that the matching is implemented, thereby implicitly ending the mechanism. In contrast, real-life matching mechanisms are not always exactly akin to one-shot games; participants are often offered the option to participate again in the mechanism, possibly by submitting a new ROL. There are often obvious reasons to do so. For example, in school choice or college admission systems it is indeed typical to see some students ending up unmatched and some schools not filling their capacities. Students may also sometimes participate in several, distinct matching markets (e.g., for private and public schools) and, since students eventually attend only one school, overlapping matching markets often entail in having wasted seats. Allowing for additional runs of a matching mechanism may give students the opportunity to improve their matches and can also mitigate the waste. In all such cases the "final" matching is not constructed at once but gradually, across several runs or periods. That is, participants may not get all their final match in the same run; some may be matched in the first run while others are matched at later runs. The objective of this paper is to offer a new framework to study such gradual matching mechanisms in the context of a many-to-one college admission problem.

The general structure of a gradual matching mechanism is thus a multi-period matching mechanism where,

(i) at each period there is a one-shot matching mechanism;

(ii) at the end of each non-terminal period each participant can either finalize his match or continue by participating in the next period's matching mechanism;

(iii) there is a unique matching that is implemented, where each participant's match is given by his last match (i.e., his match when he finalized or the last period match otherwise).

Our description of a multi-period matching mechanism deliberately mixes centralized and decentralized aspects. The calculation of the matching at each stage is centralized (it is done by the clearinghouse using the submitted ROLs), but the decisions to continue or finalize one's match are decentralized. Throughout the paper we do not address students' 
strategic decisions regarding the ROLs they submit at each period and their participation to additional periods. ${ }^{1}$ That is, we take students' submitted ROLs and periods at which they finalize their match as given. Our work is thus very much in the spirit of Gale and Shapley's original work in the sense that we investigate under which conditions a gradual matching mechanism outputs matchings that satisfy certain properties for any possible profile of ROLs submitted by the students.

There are a number of real-life instances of gradual matching mechanisms. A prominent case is the two-round New York City school choice system for middle and high schools that was in place until 2019, where both rounds consisted of running a standard school matching mechanism (see Abdulkadiroğlu et al., 2005). Students who were unassigned at the end of the first round, as well as students who were not satisfied by their first-round assignment were offered the opportunity to participate again to the school choice mechanism in a second round. Those students could put in their ROL any school that had available seats, independently of whether they included those schools in their first round ROL. ${ }^{2}$ Another example, which we analyze in more details in this paper, is the college admission mechanism used in France until 2017. ${ }^{3}$ This mechanism was matching students in three rounds and was similar in spirit to the two-round mechanism used in New York City. That is, at the end of the first and second rounds students had to decide whether to finalize their match or participate to an additional run of a "static" matching mechanism. Interestingly, for the second and third rounds students in France could only consider schools that were in their ROL in the previous round. Students were allowed, however, to modify the relative ranking of those schools. Some of the motives for allowing preference updating were because students' preferences over colleges may be affected by factors such as where their friends have been admitted at previous runs, the evolution of housing opportunities or simply additional information about colleges or career prospects. ${ }^{4}$ The New York and French cases are not the only examples. For instance, Andersson et al. (2018) comment extensively on the Swedish

\footnotetext{
${ }^{1}$ We discuss strategic incentives in Appendix B. See also Doğan and Yenmez (2018).

${ }^{2}$ The seats that were available for the second round are those that were either unfilled in the first round or made available by the students who were assigned to them but who opted for participating to the second round.

${ }^{3}$ In 2018 college admissions in France became a decentralized system.

${ }^{4}$ Antler (2015) analyzes the structure of equilibria in presence of endogenous preferences in the context of a standard one-to-one matching model where participants can be influenced by the assignments and/or preferences of others.
} 
and Turkish systems, which also propose students to participate to an additional run of a matching mechanism.

Following the French college admission example, a key assumption we make in this paper is that, at each period, students are allowed to submit a new, different ROL. However, we impose that the new ROL does not make acceptable a college that was previously declared unacceptable. ${ }^{5}$ ROL updating is modeled through a refitting rule, which describes for each student the set of permitted ROLs that can be submitted at every period.

To state our results, we maintain throughout the paper the assumption that the one-shot matching mechanism used at every period is stable. That is, the mechanism always produces an assignment satisfying the standard conditions of individual rationality, non wastefulness, and absence of justified envy. The assumption is strengthened afterward by considering the two most prominent stable mechanisms, namely the Student-Optimal Stable mechanism (SOSM) and the College-Optimal Stable mechanism (COSM). For instance, SOSM and COSM, obtained via Deferred Acceptance algorithms, are/were executed at every period of the NYC school choice system and of the French college admission system, respectively.

One of the key issues in gradual matching mechanisms is whether students can safely participate to additional runs of the matching mechanism, that is, whether participants can at each additional period somehow secure the match from their previous period. Without such a guarantee the incentives to be part of an additional run of the matching mechanism wanes, thereby undermining the motive to run a gradual matching mechanism. We capture such a guarantee with the concept of gradual safety, which simply states that a any period a participant's match is ranked weakly higher than the match of the previous period. Our first objective is to study the interplay between gradual safety and the degree of freedom left to students as they update their ROLs through the refitting rule.

A simple policy to implement gradual safety would be to alter each college's priority ranking by moving to the top the students matched to it at the previous period. Our main results show under which conditions gradual safety can be ensured without such a policy. We identify the restrictions on the refitting rules such that any gradual mechanism consistent with those restrictions is gradually safe. Those restrictions depend on the way students are assigned, at each period, by the one-shot matching mechanism. The results are thus established for three different configurations: any generic stable one-shot mechanism, SOSM

\footnotetext{
${ }^{5}$ Note that this restriction was not satisfied for the New York City school choice system.
} 
and COSM. Besides technical conditions that we do not discuss here, the main restriction we impose on any refitting rule is based on the condition of regularity (or a declination of it). The regularity condition roughly states that any college $c$ that has been ranked below one's match $v$ cannot be ranked above $v$ at the next period. Regarding colleges that are ranked above one's match, the regularity condition does not impose anything; the relative ranking of these schools can be reshuffled and any of them can be declared as unacceptable. We show that the regularity condition is necessary and sufficient for gradual safety to hold when, at each period, the matching mechanism is SOSM (Theorem 1). Our results are also used to derive by-product results in the case of gradual matching mechanisms without refitting. In those mechanisms of practical interest, each student submits his ROL only once at the first period and, from period 2 onwards, the period at which the match is finalized is the only remaining degree of freedom left to the students. Our results show how the choice of one specific one-shot mechanism, typically SOSM or COSM, may affect the benefits of participating to additional periods.

The notion of stability needs to be adapted to the context of gradual matching because agents may not all be matched at the same period. We thus introduce a concept of gradual stability, which is built upon the following principle: when a student finalizes his match he exits the mechanism, thus waiving all the claims he could have about the matchings that could arise after he left. In other words, student's claims are legitimate only for the matchings that arose while the student was participating, that is, claims will necessarily be backward looking. Although gradual stability is clearly more demanding than the standard, static stability concept, we show that under the regularity condition gradual stability is in fact equivalent to gradual safety together with (static) stability (Theorem 2).

We also consider in the paper the case of withdrawals, that is, situations where students may renounce to their assignments after having finalized them (but before the last period). The presence of withdrawals introduces a minor complication because if the newly vacant seats are made available to the students for the next periods colleges' capacities are no longer decreasing over periods. Nevertheless, we show that our main results are still valid in this environment.

Finally, we use our framework to examine the college admission system that was in place in France until 2017, which corresponds to a three-period gradual matching mechanism. ${ }^{6}$

\footnotetext{
${ }^{6}$ By college admissions we mean the allocation of high school students to any institution of higher education to pursue undergraduate studies, which covers many different kinds of institutions in France.
} 
Students in France were allowed to update their ROLs across periods, and for each of the three periods the static matching mechanism used the Deferred Acceptance algorithm with colleges proposing. It is easily shown that the refitting rule used in France satisfies a strong version of regularity, thereby implying that it is a gradually safe and gradually stable mechanism.

The rest of the paper is organized as follows. We outline in Section 2 the basic college admission model we consider in the paper, which we extend in Section 3 by defining gradual matching problems. Sections 4 and 5 contain the main results identifying gradually safe mechanisms and gradual stability, respectively. We address in Section 6 the issue of students' withdrawals. In Section 7 we apply our results to the French system for college admissions and we conclude in Section 8. In Appendices A and B we offer a short discussion on gradual safety and strategic behavior, respectively. Proofs are relegated to Appendix C.

\section{Literature}

With respect to the existing literature, gradual matching problems differ substantially from dynamic matching problems, which generally consist of situations where participants, and thus matching opportunities, arrive sequentially, and/or where agents may possibly "consume" different matches at different periods. For those models, agents' preferences are usually defined over sequences of matches. Dynamic problems with irreversible matches can be found in Doval (2019), Baccara et al. (2020), Bloch and Cantala (2017) or Akbarpour et al. (2020). Models with limited commitment, i.e., where agents can be matched multiple times across periods are studied by Combe et al. (2018), Kadam and Kotowski (2018a,b), Kotowski (2019), Kennes et al. (2014, 2019) and Pereyra (2013).

In contrast, in gradual matching problems all participants are present at the outset and, like for static matching problems, there is only one matching that is implemented. The dynamic nature of gradual matching mechanism comes rather from the fact that the matching mechanism is run several times, assigning participants progressively. This class of problems has been studied in separate analytical frameworks by Andersson et al. (2018), Manjunath and Turhan (2016), Doğan and Yenmez (2018), Dur and Kesten (2019) and Westkamp (2012). ${ }^{7}$ Doğan and Yenmez (2018) consider a gradual mechanism similar to ours, but their

\footnotetext{
${ }^{7}$ Gradual matching also arises when participants can contract bilaterally before the job openings, as identified first by Roth (1984) and documented by Roth (1991) and Kagel and Roth (2000) in different labor
} 
work differs from ours in several important aspects. First, they only consider two-period gradual mechanisms and, second, they study the equilibrium outcomes of their mechanism. That is, they do not study like us the outcome properties for all possible ROLs submitted by the students (we comment more on their findings in Appendix B). The other aforementioned contributions are not directly comparable to ours. A major difference between these works and ours is that, contrary to our modeling, subsequent periods of matching are designed to make available new seats or positions on the side of universities/colleges/schools. It can be either seats in private schools (versus public schools in the first round) as in Andersson et al. (2018) or seats in other distinct enrollment procedures as in Manjunath and Turhan (2016), or positions non tenured any more after the first period as in Dur and Kesten (2019), or seats released purposely only in the second period as in Westkamp (2012).

Participants in a gradual matching mechanism face two strategic issues: a preference revelation decision at each period and whether to finalize or participate to the next period's matching mechanism. Those two issues are obviously connected; strategic choices in terms of preference revelation may affect one's match, which in turn may affect one's decision about participating to an additional run of the matching mechanism. Doğan and Yenmez (2018) examine precisely the students' behavior in that context and identify the profiles of priorities and capacities of colleges under which an additional period of matching benefits students in equilibrium. Regarding incentives, we show in Appendix B that gradual mechanisms are not strategyproof, even if a strategyproof mechanism is used at each period. Papers discussing the non-existence of strategyproofness in related frameworks include the studies of Dur and Kesten (2019) or Andersson et al. (2018). This feature is also present in the case of multi-period matching with long-lived participants and limited commitment, as shown by Kennes et al. (2014, 2019) and Pereyra (2013). Similar opportunities for manipulations were already identified by Kesten (2010) for the Efficient Adjusted Deferred Acceptance mechanism (EADAM) that is based on the removal of specific students during the rounds of the static Deferred Acceptance algorithm.

markets. Halaburda (2010) and Echenique and Pereyra (2016) define general matching models to address this issue. Avery and Levin (2010) consider decentralized admissions with early periods of admissions, used by students to signal their interest to colleges. 


\section{Preliminaries}

We consider in this paper the problem of matching students to colleges in the limit of their capacities. Throughout the paper we will consider a finite set $I$ of students and a finite set $C$ of colleges.

Each college $c \in C$ is endowed with a fixed capacity of seats $q_{c} \geq 0$, and a strict priority ordering $P_{c}$ over the set of students. We write $i P_{c} j$ to say that that student $i$ has higher priority than student $j$ at college $c$.

Each student $i \in I$ has a strict rank order list (ROL) $P_{i}$ over the colleges and the option of remaining unassigned, i.e., $P_{i}$ is a linear ordering over $C \cup\{\emptyset\}$, with $c P_{i} c^{\prime}$ to denote that college $c$ is ranked above college $c^{\prime}$ by student $i$. A college $c$ is acceptable for a student $i$ under the ROL $P_{i}$ if $c$ is ranked above being unmatched, i.e., $c P_{i} \emptyset$. We sometimes use the following notation $P_{i}=\left[c_{1}, c_{2}, \ldots, c_{j}, \emptyset, \ldots\right]$ to denote that student $i$ 's first choice is $c_{1}$, his second choice $c_{2}$ (i.e., $c_{1} P_{i} c_{2}$ ), and so on. Given a ROL relation $P_{i}$ we denote by $R_{i}$ the weak relation associated to it, i.e., $v R_{i} v^{\prime} \Leftrightarrow v P_{i} v^{\prime}$ or $v=v^{\prime}$. Hereafter we will often drop the subscript when referring to a ROL, i.e., we will write $P$ to denote a linear ordering over $C \cup\{\emptyset\}$.

\section{A college admission problem is a 5-tuple}

$$
\Gamma=\left(I, C,\left(P_{c}, q_{c}\right)_{c \in C},\left(P_{i}\right)_{i \in I}\right)
$$

We denote by $\mathcal{P}$ the set of all ROLs over $C \cup\{\emptyset\}$. Given a ROL $P \in \mathcal{P}$, we denote also by $A_{P}$ the acceptability set, i.e., $A_{P}:=\{c \in C: c P \emptyset\}$.

A matching for a college admission problem $\left(I, C,\left(P_{c}, q_{c}\right)_{c \in C},\left(P_{i}\right)_{i \in I}\right)$ is a mapping $\mu$ : $I \cup C \rightarrow 2^{I} \cup C$ such that, for each $i \in I$ and each $c \in C$,

- $\mu(i) \in C \cup\{\emptyset\}$,

- $\mu(c) \in 2^{I}$,

- $\mu(i)=c$ if, and only if, $i \in \mu(c)$,

- $|\mu(c)| \leq q_{c}$.

For $v \in C \cup I$, we call $\mu(v)$ agent $v$ 's assignment. For $i \in I, \mu(i)=c$ for some $c \in C$ means that student $i$ is matched to college $c$ under $\mu, \mu(i)=\emptyset$ means then student $i$ is not matched to any college under $\mu$. 
Students' ROLs can be straightforwardly extended to ordering over matchings. We say that matching $\mu$ is ranked above matching $\mu^{\prime}$ for student $i$ if his assignment under $\mu$ is ranked above his assignment under $\mu^{\prime}$. Formally, $\mu P_{i} \mu^{\prime}$ if $\mu(i) P_{i} \mu^{\prime}(i)$, and $\mu R_{i} \mu^{\prime}$ if $\mu P_{i} \mu^{\prime}$ or $\mu(i)=\mu^{\prime}(i)$. If $\mu R_{i} \mu^{\prime}$ we say $\mu$ is ranked weakly above $\mu^{\prime}$ by student $i$.

A matching is stable if each student is matched to an acceptable college and all the colleges ranked in his ROL above his assignment have exhausted their capacities with students that all have higher priority. Formally, a matching $\mu$ is stable for a college admission problem $\Gamma$ if $\mu$ is a matching for $\Gamma$ and

(a) it is individually rational, i.e., for all $i \in I, \mu(i) R_{i} \emptyset$;

(b) it is non wasteful, i.e., for all $i \in I$ and all $c \in C, c P_{i} \mu(i)$ implies $|\mu(c)|=q_{c}$;

(c) there is no justified envy,i.e., for all $i, j \in I$ with $\mu(j)=c \in C, c P_{i} \mu(i)$ implies $j P_{c} i .^{8}$

It is well known that for any college admission problem the set of stable matchings is non-empty (Gale and Shapley, 1962). A matching mechanism $\varphi$ maps college admission problems to matchings. It is stable if for each college admission problem $\Gamma, \varphi(\Gamma)$ is stable. Given a college admission problem $\Gamma$, we denote the student-optimal (resp. pessimal) stable matching by $S O S M(\Gamma)$ (resp. $C O S M(\Gamma)$ ), obtained through the implementation of the student-proposing (resp. college-proposing) Deferred Acceptance algorithm. ${ }^{9}$ The shorthands SOSM and COSM denote the associated mechanisms.

\section{Gradual matching}

\subsection{An illustrative example}

The next example aims at capturing some of the key aspects of a mechanism that integrates additional periods of assignment.

Example 1 Consider a college admission problem with five students and four colleges, each with a capacity equal to one. The ROLs submitted by the students and the priorities of the colleges are described in Table 1. We consider the matching of students during two

${ }^{8}$ Notice that the way we define stability implicitly assumes that colleges priorities over sets of students are responsive (Roth, 1985).

${ }^{9}$ See Gale and Shapley (1962) and Abdulkadiroğlu and Sönmez (2003). 
periods. At each period SOSM is computed according to the college admission problem at that period.

\begin{tabular}{ccccc}
\multicolumn{5}{c}{ Students } \\
\hline$P_{i_{1}}$ & $P_{i_{2}}$ & $P_{i_{3}}$ & $P_{i_{4}}$ & $P_{i_{5}}$ \\
\hline$c_{2}$ & $c_{1}$ & $c_{1}$ & $c_{3}$ & $c_{1}$ \\
$c_{1}$ & $c_{3}$ & $c_{2}$ & $c_{4}$ & $c_{4}$ \\
$\emptyset$ & $\emptyset$ & $\emptyset$ & $\emptyset$ & $\emptyset$
\end{tabular}

\begin{tabular}{cccc}
\multicolumn{4}{c}{ Colleges } \\
\hline$P_{c_{1}}$ & $P_{c_{2}}$ & $P_{c_{3}}$ & $P_{c_{4}}$ \\
\hline$i_{1}$ & $i_{2}$ & $i_{2}$ & $i_{1}$ \\
$i_{2}$ & $i_{3}$ & $i_{1}$ & $i_{2}$ \\
$i_{3}$ & $i_{1}$ & $i_{3}$ & $i_{3}$ \\
$i_{4}$ & $i_{4}$ & $i_{4}$ & $i_{4}$ \\
$i_{5}$ & $i_{5}$ & $i_{5}$ & $i_{5}$
\end{tabular}

Table 1: A college admission problem

At period 1 we obtain the matching $\mu^{1}$ (indicated by the boxes in Table 1 ),

$$
\mu^{1}\left(i_{1}\right)=c_{1}, \mu^{1}\left(i_{2}\right)=c_{3}, \mu^{1}\left(i_{3}\right)=c_{2}, \mu^{1}\left(i_{4}\right)=c_{4}, \mu^{1}\left(i_{5}\right)=\emptyset .
$$

We now consider three possible scenarios at period 2. These scenarios illustrate the different ways the gradual matching of students can differ from the outcome of the classic one-period matching mechanism. For simplicity, we assume that students $i_{1}$ and $i_{3}$ will always participate to period 2 matching mechanism, submitting the same ROL in both periods.

\section{- First scenario}

Due to scheduling constraints student $i_{2}$ does not take part to the second round. He thus finalizes his first period match with $c_{3}$. That is, $i_{2}$ is removed from the problem and is permanently matched to $c_{3}$. In the second period the other students submit again the same ROLs and obtain a new match given by $\mu^{2}$,

$$
\mu^{2}\left(i_{1}\right)=c_{2}, \mu^{2}\left(i_{3}\right)=c_{1}, \mu^{2}\left(i_{4}\right)=c_{4}, \mu^{2}\left(i_{5}\right)=\emptyset
$$

Students $i_{1}$ and $i_{3}$ are strictly better off in the second period. That is, even if students do not update their ROLs the very fact that some students finalize their match before others can affect the match of the students who participate to an additional matching period. Note that the final matching (for the five students) is not stable since $i_{2}$ has a justified envy at $c_{1}$. That is, gradual matchings may not be stable even if at each 
period a stable matching mechanism is used to match students and colleges. This is not surprising given the lack of consistency of the stable matching operator in gradual matching problems. ${ }^{10}$ This fact motivates a conceptual reconsideration of the stability concept that accounts for the sequentiality of the procedure, which we do in Section 5.

\section{- Second scenario}

Student $i_{2}$ remains among the active students. But in the second period he submits a new ROL, $\left[c_{2}, c_{1}, c_{3}, \emptyset\right]$. All other things remaining equal, the students obtain a new match, $\widehat{\mu}^{2}$ given by

$$
\widehat{\mu}^{2}\left(i_{1}\right)=c_{1}, \widehat{\mu}^{2}\left(i_{2}\right)=c_{2}, \widehat{\mu}^{2}\left(i_{3}\right)=\emptyset, \widehat{\mu}^{2}\left(i_{4}\right)=c_{3}, \widehat{\mu}^{2}\left(i_{5}\right)=c_{4} .
$$

Here, the change of $i_{2}$ 's ROL is harmful for student $i_{3}$ who gets no seat. In the remainder, we will identify conditions on gradual matching mechanisms that never produce such outcomes. We will see later in the paper that this requires to control precisely to which extent the students can resubmit new ROLs (see Section 4).

\section{- Third scenario}

A good outside option for student $i_{2}$ emerges at the very end of the first period. He withdraws from his assignment $c_{3}$ and he is removed from the problem. ${ }^{11}$ Here, the new vacant seat after the first period is put back into the pool of available seats so that all the active students can possibly take advantage of $i_{2}$ 's withdrawal. All other things remaining equal, the final matching is

$$
\widetilde{\mu}^{2}\left(i_{1}\right)=c_{2}, \widetilde{\mu}^{2}\left(i_{3}\right)=c_{1}, \widetilde{\mu}^{2}\left(i_{4}\right)=c_{3}, \widetilde{\mu}^{2}\left(i_{5}\right)=c_{4}
$$

We account for withdrawals in Section 6.

\subsection{The base model}

We consider a finite number of periods $t=1, \ldots, T$. A sequence of $T$ college admission problems $\Gamma^{1}, \Gamma^{2}, \ldots, \Gamma^{T}$ is nested if for each $\Gamma^{t}=\left(I^{t}, C^{t},\left(P_{c}^{t}, q_{c}^{t}\right)_{c \in C},\left(P_{i}^{t}\right)_{i \in I}\right), t=1, \ldots, T$, the following properties hold:

\footnotetext{
${ }^{10}$ See Ergin (2002).

${ }^{11}$ If the second period were a secondary scrambling session available only to the unmatched students, $c_{3}$ should be assigned to the student $i_{5}$ who is unmatched. But since $c_{3}$ is not acceptable for $i_{5}$ the seat would remain vacant under SOSM.
} 
- $I^{t+1} \subseteq I^{t}$ for each $1 \leq t<T$;

- $C^{t}=C$ for each $1 \leq t \leq T$;

- For each college $c \in C, P_{c}^{t}:=\left.P_{c}^{1}\right|_{I^{t}}$ for each $2 \leq t<T$, where $\left.P_{c}^{1}\right|_{I^{t}}$ is the restriction of $P_{c}^{1}$ to the set of students $I^{t}$.

A nested sequence of college admission problems does not necessarily consist of identically repeated college admission problems for two reasons.

First, some students may leave the problem before the final period $T$. We will often refer to the students in $I^{t}$ for some given $t$ as the active students (at period $t$ ). Given a sequence $\left(\Gamma^{t}\right)_{t \leq T}$, for each student $i \in I^{1}$, we denote by $t_{i}$ the latest period at which the student is in the set of students, i.e., $t_{i}=\operatorname{argmax}_{1 \leq t \leq T}\left\{i \in I^{t}\right\}$, and thus say that $i$ finalizes his match at period $t_{i}$.

A second reason, and perhaps more interesting for us, is that students may have different ROLs from one period to the other, i.e., for some $t \neq t^{\prime}$ we may have $P_{i}^{t} \neq P_{i}^{t^{\prime}}$ for some student $i \in I^{t}, I^{t^{\prime}}$. Recall, however, that colleges' priorities over active students are fixed across periods.

To each nested sequence of problems $\left(\Gamma^{t}\right)_{t \leq T}$ we can naturally associate a sequence of matchings $\left(\mu^{t}\right)_{t \leq T}$. A sequence $\left(\Gamma^{t}, \mu^{t}\right)_{t \leq T}$ is feasible if $\left(\Gamma^{t}\right)_{t \leq T}$ is a nested sequence of college admission problems and $\mu^{t}$ is a matching for $\Gamma^{t}$, for each $1 \leq t \leq T$. A gradual outcome describes the feasible sequences of problems and matchings that are mutually consistent across periods.

Definition 1 A feasible sequence $\left(\Gamma^{t}, \mu^{t}\right)_{t \leq T}$ is a gradual outcome if for each $1 \leq t<T$, and each college $c \in C$,

$$
q_{c}^{t+1}=\left(q_{c}^{t}-\left|\mu^{t}(c)\right|\right)+\left|\left\{i \in I^{t+1}: \mu^{t}(i)=c\right\}\right|
$$

For convenience, whenever there is no confusion we will use the shorthand outcome instead of gradual outcome.

Equation (1) links each problem in a sequence with the match of the previous period. For every college, the capacity at each college consists of adding the number of seats previously left vacant to the number of seats assigned previously to students still active at the current period. Equivalently, the seats assigned to the students who left the problem at a previous period are deducted from the capacity the college had at the previous period. 
An outcome $\left(\Gamma^{t}, \mu^{t}\right)_{t \leq T}$ implicitly defines a matching $\nu$ of particular interest,

$$
\nu(i)=\mu^{t_{i}}(i), \quad \forall i \in I .
$$

For each student $i, \nu(i)$ describes the assignment of the student $i$ at the time he leaves the problem, i.e., his finalized match. Note that, by construction, $\nu$ is a matching for the initial problem $\Gamma^{1}$. The matching $\nu$ is called the gradual matching of $\left(\Gamma^{t}, \mu^{t}\right)_{t \leq T}$.

Equations (1) and (2) capture together what makes the matching of students gradual: some students "leave with their assignment" at a given period and, by doing so, may affect the assignments of remaining students that can arise at later periods.

\subsection{Refitting rules}

The scenarios developed in Section 3.1 show that we need to impose some restrictions on which ROLs students can submit in order to guarantee that outcomes satisfy certain properties. We do this using the concept of refitting rule, which delimits, for each period (except for the first period) each active student's domain of permitted ROLs. We will see that it is sufficient to have a refitting rule depending on two parameters only: the ROL used by a student and the match of that student at the previous period.

Definition 2 A refitting rule is a set-valued mapping $F: \mathcal{P} \times(C \cup\{\emptyset\}) \rightrightarrows \mathcal{P}$.

We will use a refitting rule as follows. Let $i$ be student $i$ who participated at some period $t$ to the matching mechanism with the ROL $P_{i}$ and let $v$ be $i$ 's match at that period. If $i$ is still an active student in period $t+1$ then the only ROLs that can be used in that period must belong to the set $F\left(P_{i}, v\right)$. In a refitting set $F(P, v)$ we call $v$ the proposal.

Throughout the paper we assume that any refitting rule satisfies the following two elementary properties,

(i) For every $(P, v) \in \mathcal{P} \times(C \cup\{\emptyset\})$, if $v \in A_{P}$ then $A_{P^{\prime}} \neq \emptyset$ for some $P^{\prime} \in F(P, v)$;

(ii) For every $(P, v) \in \mathcal{P} \times(C \cup\{\emptyset\})$ and $P^{\prime} \in F(P, v)$, it holds that $A_{P^{\prime}} \subseteq A_{P}$.

We denote by $\mathcal{F}$ the set of all refitting rules satisfying conditions (i) and (ii) above. Condition (i) says that if a college is proposed to a student at some period then at least one college is acceptable in some permitted ROL for the next period. Condition (ii) states that a student cannot add a new college in his acceptable set at any permitted ROL for the next period, 
i.e., the acceptability sets are nested across time. ${ }^{12}$ This condition is for instance satisfied in the French college admissions system, where students can only remove colleges from their former acceptable sets from one period to another. However, condition (ii) is not satisfied for instance in so-called "scramble markets," where additional runs are only open for colleges that haven't filled their capacities. In such cases students rank new colleges. This case is studied by Andersson et al. (2018). This assumption is also violated in dynamic matching problems where new colleges are added to the problem at some subsequent periods (e.g., see Westkamp, 2012).

The possibility to resubmit a new ROL has a bite only if the refitting is a set-valued mapping, that is, when the student can choose among at least two ROLs. Indeed, if the rule is single-valued then the outcome are fully determined by the first period's ROLs and players' finalization periods, $\left(t_{i}\right)_{i \in I}{ }^{13}$

We now define two conditions that will play a key role in our analysis.

Definition 3 The rule $F \in \mathcal{F}$ is regular if for every $(P, v) \in \mathcal{P} \times(C \cup\{\emptyset\})$ and $c \in C \backslash\{v\}$,

$$
v P c \Rightarrow v P^{\prime} c \text { if } c \in A_{P^{\prime}}, \quad \forall P^{\prime} \in F(P, v) .
$$

A regular refitting rule imposes that any college $c$ ranked below the proposal $v$ must still be ranked below the proposal at the next period whenever college $c$ is declared acceptable at the next period. A simple regular single-valued rule is the identity mapping $F^{I D}$ : for every $(P, v) \in \mathcal{P} \times(C \cup\{\emptyset\})$,

$$
F^{I D}(P, v)=\{P\}
$$

Another example is the following regular set-valued refitting rule: for every $(P, v) \in \mathcal{P} \times(C \cup$ $\{\emptyset\})$,

$$
F(P, v)=\left\{P^{\prime} \in \mathcal{P}: A_{P^{\prime}} \subseteq A_{P} ; v \in A_{P} \Rightarrow v \in A_{P^{\prime}} ; \forall c, c^{\prime} \in A_{P^{\prime}}, c P c^{\prime} \Rightarrow c P^{\prime} c^{\prime}\right\} .
$$

That is, students maintain every acceptable proposal in the next ROL and are allowed to remove other colleges, without changing the relative ranking among acceptable colleges.

Definition 4 The rule $F \in \mathcal{F}$ is strongly regular if for every $(P, v) \in \mathcal{P} \times(C \cup\{\emptyset\})$ and $c \in C \backslash\{v\}$

$$
v P c \Rightarrow \emptyset P^{\prime} c, \quad \forall P^{\prime} \in F(P, v) .
$$

\footnotetext{
${ }^{12}$ The role of that assumption appears more clearly in the next section.

${ }^{13} \mathrm{We}$ analyze single-valued refitting rules in Section 4.3.
} 
Strong regularity requires any college ranked below the current period assignment to be unacceptable in the next period's permitted ROLs. A simple strongly regular single-valued rule is the truncation mapping $F^{T R}$, for instance, if $P_{i}=\left[c_{1}, c_{2}, v, \ldots, c_{k}, \emptyset, \ldots\right]$ then $F^{T R}\left(P_{i}, v\right)$ is the singleton set $\left\{\left[c_{1}, c_{2}, v, \emptyset, \ldots\right]\right\}$. Note that the identity mapping is not a strongly regular rule.

A final simple instance of refitting rule is the MT rule, proposed by Manjunath and Turhan (2016). As they consider the assignments of students in separate centralized enrollment systems, Manjunath and Turhan (2016) define a new class of matching mechanism based on a full fledged refitting rule, which works as follows. At every period, the permitted ROLs are defined, in each enrollment system, accordingly to a bimodal rule. The new ROL is either the previous ROL truncated below the previous student's assignment or the previous ROL truncated above the previous student's assignment (which can be $\emptyset$ in both cases), truncating below or above is determined by the assignments the student obtains in the other systems (he keeps the best one). The MT rule $F^{M T}$ can be formally defined as follows (in each enrollment system): for every $(P, v) \in \mathcal{P} \times(C \cup\{\emptyset\})$ with $P=\left[c_{1}, c_{2}, \ldots, c_{j}, v, c_{k}, \ldots\right]$,

$$
F^{M T}(P, v)=\left\{\left[c_{1}, c_{2}, \ldots, c_{j}, v, \emptyset, \ldots\right],\left[c_{1}, c_{2}, \ldots, c_{j}, \emptyset, \ldots\right]\right\} .
$$

The rule $F^{M T}$ belongs to $\mathcal{F}$ and has two interesting features in our context. First, it is a meaningful instance of a non single-valued refitting rule. Second, $F^{M T}$ is obviously strongly regular.

\subsection{Gradual matching mechanisms}

We define in this section gradual matching mechanisms. Roughly speaking, such mechanisms work as follows. We start with a college admission problem where each student participates to a matching mechanism by submitting a ROL. Once the matching is announced students can either finalize their match or participate to another matching mechanism. The set of students that remain active, together with their new ROLs (permitted under the refitting rule $F$ ) define the next college admission problem. Repeating the procedure for $T-1$ additional periods yields a gradual outcome as defined in Section 3.2. Our main objective is to study the mechanisms that produce gradual outcomes.

To distinguish between multi-period mechanisms and the (more traditional) matching mechanism that matches students at each period we will refer to this latter as a spot 
mechanism, i.e., a mechanism for a standalone college admission problem.

In an outcome the ROLs submitted by a student $i$ consist of a list $\mathbf{P}_{i}$ of all the submitted ROLs from period 1 until the period at which the student finalized his match, $t_{i}$. So, $\mathbf{P}_{i}=\left(P_{i}^{t}\right)_{1 \leq t \leq t_{i}}$. For any nonempty set $J \subseteq I$, we denote by $\mathbf{P}_{J}$ the profile of submitted ROLs of by students in $J, \mathbf{P}_{J}=\left(\mathbf{P}_{i}\right)_{i \in J}$. Note that since students may not finalize their match at the same period the lists of two students may not have the same length. Hence, a profile $\mathbf{P}_{I}$ implicitly includes the period at which each student finalized his match, which is simply equal to the length of his list in $\mathbf{P}_{I}$.

Besides the spot mechanism and the refitting rule, the initial problem $\Gamma^{1}$ and the sequence of submitted ROLs, $\mathbf{P}_{I}$, thus contain all the relevant information that is needed to construct outcomes.

A gradual matching mechanism maps college admission problems and sequences of submitted ROLs to outcomes. A generic gradual matching mechanism is denoted by $\mathcal{M}_{F}^{\varphi}$, where $\varphi$ is the spot college admission mechanism and $F$ is the refitting rule. For each problem $\boldsymbol{\Gamma}=\left(I^{1}, C,\left(P_{c}, q_{c}^{1}\right)_{c \in C}, \mathbf{P}_{I^{1}}\right), \mathcal{M}_{F}^{\varphi}(\boldsymbol{\Gamma})$ is the outcome $\left(\Gamma^{t}, \mu^{t}\right)_{t \leq T}$ such that

(i) $\mu^{t}=\varphi\left(\Gamma^{t}\right)$ for each period $t=1, \ldots, T$;

(ii) $P_{i}^{t} \in F\left(P_{i}^{t-1}, \mu^{t-1}(i)\right)$ for all $i \in I^{t}$, for each period $t=2, \ldots, T$.

Remark 1 Our description of a multi-period college admission mechanism deliberately mixes centralized and decentralized aspects. The calculation of the matching at each period is centralized (it is done by the clearinghouse using the submitted ROLs), but whether students remain active or finalize their match is decentralized in the sense that is not part of the matching mechanism. A fully centralized version would require students to also submit schedules of ROLs and accept/decline decisions for each period (possibly conditional on the matching obtained at a given period).

Also, note that we are agnostic about students' decisions regarding the period at which they finalize their match. We take students' sequences of submitted ROLs (and thus the period at which they exit the mechanism) as given, our only concern being what are the conditions on the mechanism (the spot matching mechanism and the refitting rule) that guarantee some properties for any input (students' sequences of submitted ROLs). 


\section{Gradually safe mechanisms}

We restrict our attention to the class of gradual matching mechanisms for which the spot mechanism is assumed to be stable, as it is often the case in practice or advocated in the matching literature.

Example 1 (scenario 2) already shows that gradual matching mechanisms do not always guarantee that participating in an additional round cannot make the students worse off. An elementary property one would thus require for a gradual matching mechanism is to somehow guarantee each student the spot at the college they have been assigned at the previous period. There are several ways to implement such a property.

A first approach is, at the beginning of each period, to lock students' seats at their former assignments. This is clearly not a satisfactory solution because it would eventually entail in wasteful assignments (and also, at each period only only colleges that did not filled their capacities would be available). A second approach would be instead to modify, at each period $t \geq 2$, each college's $c \in C$ priority ranking by moving to the top $q_{c}^{t}$ position all the active students who were matched to $c$ at the period $t-1$. This modified matching mechanism can be viewed as a natural option to guarantee participants their former positions for problems like the re-assignment of teachers with tenured positions, as studied by Combe et al. (2018) and Pereyra (2013). Such seniority based rules are fully adapted to environments like teachers' assignment problems, where agents do "consume" their matches at every period and inherit a seniority priority at the end of each period (say, after one year). However, assigning property rights (and thus modifying priorities) across periods is not appropriate here since we consider a procedure that is delimited to only one enrollment campaign. Our framework is also adapted for situations where priority rankings of colleges or schools cannot or should not be modified throughout the execution of the matching mechanism. ${ }^{14}$

Our approach to guarantee students their former assignments takes a different route and requires instead that at each period in which a student is active (except the first one), he is matched to a college that is ranked weakly higher in the current period's ROL. Under such a property, the previous period's match would play the role of a safety college. Our objective in this section is to identify refitting rules such that this is indeed the case, without the need

\footnotetext{
${ }^{14}$ Such seniority rules can also generate confusion and discontentment among participants when they are regularly updated about their rank in each college's priority ranking. Take the case of a college $c$ such that $P_{c}: i_{1}, i_{2}, i_{3}$, and assume that $i_{3}$ is matched to $c$ in the first period. In the second period $c^{\prime}$ priority ordering would be $P_{c}^{2}: i_{3}, i_{1}, i_{2}$, i.e., students $i_{1}$ and $i_{2}$ would see their rank going down from one period to the next.
} 
to alter colleges' priorities.

Definition 5 An outcome $\left(\Gamma^{t}, \mu^{t}\right)_{t \leq T}$ is gradually safe if, for each $2 \leq t \leq T$ and $i \in I^{t}$,

$$
\mu^{t} R_{i}^{t} \mu^{t-1}
$$

Let $\varphi$ and $F \in \mathcal{F}$ be a spot mechanism and a refitting rule, respectively. The gradual matching mechanism $\mathcal{M}_{F}^{\varphi}$ is gradually safe if, for every $\boldsymbol{\Gamma}=\left(I^{1}, C,\left(P_{c}, q_{c}^{1}\right)_{c \in C}, \mathbf{P}_{I^{1}}\right)$, the outcome $\mathcal{M}_{F}^{\varphi}(\boldsymbol{\Gamma})$ is gradually safe.

Remark 2 Gradual safety is a backward looking concept in the sense that the previous assignment is appraised using the current ROL. An alternative approach would be to have a forward looking approach, that is, appraising the next period's assignment wit the current ROL. Formally, for each $2 \leq t \leq T$ and $i \in I^{t}$,

$$
\mu^{t} R_{i}^{t-1} \mu^{t-1}
$$

Considering condition (6) instead of condition (5) turns out to be without difficulty. We indeed show in Appendix A that, under a mild condition, Eq. (5) implies Eq. (6).

It is relatively straightforward to see that condition (ii) in the definition of a refitting rule (the sets of acceptable colleges are nested across periods) is a minimal requirement to guarantee gradual safety. Consider for instance the case of two students, $i_{1}$ and $i_{2}$, and two colleges $c_{1}$ and $c_{2}$ with $i_{2} P_{c_{1}} i_{1}$ and $i_{2} P_{c_{2}} i_{1}$, and $\varphi$ is a stable matching mechanism. Suppose that in the first period $i_{1}$ and $i_{2}$ submit $P_{i_{1}}:\left[c_{1}, \emptyset\right]$ and $P_{i_{2}}=\left[c_{2}, \emptyset\right]$. Both students participate in the second period mechanism. Student $i_{1}$ submit the same ROL as in the first period but $i_{2}$ submits $\left[c_{1}, \emptyset\right]$. Student $i_{1}$ then ends up being unmatched, i.e., gradual safety does not hold.

\subsection{The case of stable spot mechanisms}

We first consider the case where the spot mechanism is any stable mechanism (i.e., not necessarily SOSM or COSM).

Proposition 1 Let $\varphi$ be a stable spot mechanism. If $\mathcal{M}_{F}^{\varphi}$ is gradually safe for some $F \in \mathcal{F}$ then $F$ is regular. 
Proof See Appendix C.

The next example shows that the converse implication does not hold. If $F$ is the identity rule (hence regular) then gradual safety may fail when the spot mechanism $\varphi$ is stable.

Example 2 There are three colleges and three students. The capacity of each college is equal to one. There are two periods. The first period college admission problem is described in Table 2. At period 1 there is a unique stable matching, $\mu^{1}$, indicated by the boxes: $\mu^{1}\left(i_{1}\right)=c_{1}, \mu^{1}\left(i_{2}\right)=c_{2}$ and $\mu^{1}\left(i_{3}\right)=c_{3}$. Suppose that $i_{1}$ and $i_{2}$ remain active in the second period while $i_{3}$ finalizes his match at the end of the first period. ${ }^{15}$ Under the identity rule, students' ROLs are again $P_{i_{1}}^{1}$ and $P_{i_{2}}^{1}$ at period 2. Since the seat at $c_{3}$ is already assigned to $i_{3}, q_{c_{3}}^{2}=0$. Suppose that $\varphi$ selects the matching $\mu^{2}$ defined by $\mu^{2}\left(i_{1}\right)=c_{2}$ and $\mu^{2}\left(i_{2}\right)=c_{1}$. That matching is stable for the second period problem. Clearly the outcome is not gradually safe.

\begin{tabular}{ccccccc}
$P_{i_{1}}^{1}$ & $P_{i_{2}}^{1}$ & $P_{i_{3}}^{1}$ & & $P_{c_{1}}$ & $P_{c_{2}}$ & $P_{c_{3}}$ \\
\hline$c_{1}$ & $c_{2}$ & $c_{3}$ & & $i_{2}$ & $i_{1}$ & $i_{1}$ \\
$c_{3}$ & $c_{1}$ & & & $i_{1}$ & $i_{2}$ & $i_{3}$ \\
$c_{2}$ & & & & $i_{3}$ & $i_{3}$ & $i_{2}$
\end{tabular}

Table 2: A college admission problem

Nevertheless, the sufficient condition holds if one restricts the refitting rule to being strongly regular, instead of regular.

Proposition 2 Let $\varphi$ be a stable spot mechanism. If $F \in \mathcal{F}$ is strongly regular then $\mathcal{M}_{F}^{\varphi}$ is gradually safe.

Proof See Appendix C.

\footnotetext{
${ }^{15}$ Recall that we are agnostic about students' decisions, i.e., what triggers them to finalize their match a given period or remain active at the next period. The fact that $i_{1}$ and $i_{2}$ do not finalize their match when getting their top choices makes the example simple; it is an easy matter to embed the same example in a larger market (with more students and colleges) such that the proposals $c_{1}$ and $c_{2}$ are not any more the top choices of $i_{1}$ and $i_{2}$, respectively.
} 
A straightforward corollary can be obtained if one looks at the $M T$ rule defined in Section 3.3. ${ }^{16}$

Corollary 1 Let $\varphi$ be a stable spot mechanism. It holds that $\mathcal{M}_{F^{M T}}^{\varphi}$ is gradually safe.

\subsection{The case of SOSM and COSM}

Until now we have only required the spot mechanism to be any stable mechanism. Example 2 illustrates how the choice of a specific stable spot mechanism may have incidence on whether the gradual safety property holds. In period 2 , the resulting matching $\mu^{2}$, where $\mu^{2}\left(i_{1}\right)=$ $c_{2}$ and $\mu^{2}\left(i_{2}\right)=c_{1}$, corresponds to the case where $\varphi=C O S M$. Hence, the regularity assumption is not sufficient to obtain gradual safety under COSM. On the contrary, if $\varphi=$ $S O S M$ then the assignments of two remaining students are unchanged, i.e., the outcome is gradually safe. This observation may suggest that the required restrictions on the refitting rule differ depending on the choice of the spot mechanism. Our next results precisely address this issue.

Theorem $1 \mathcal{M}_{F}^{S O S M}$ is gradually safe if, and only if, $F$ is regular.

Proof The proof of the only if part is given by Proposition 1. The proof of the if part is in Appendix C.

With respect to COSM, we deduce the following result from Proposition 2.

Proposition 3 If $F \in \mathcal{F}$ is strongly regular then $\mathcal{M}_{F}^{C O S M}$ is gradually safe.

To get the converse implication we need to impose more structure on the refitting rules, requiring that refitting rules satisfy a neutrality and an independence of irrelevant colleges conditions. The neutrality property simply states that refitting rules do not depend on college's labels. The other property requires that if for some ROL $P$ and proposal $c_{1}$ a school $c_{2}$ is acceptable for some permitted $P^{\prime}$, then it is also the case for any other ROL that does not alter the relative ranking of the same proposal and $c_{2}$.

\footnotetext{
${ }^{16}$ The proof is omitted since it simply relies on the fact that $F^{M T}$ is strongly regular. Without entering into the details of their model, Corollary 1 can be used to show that the so-called $L$-iterated child optimal rule defined by Manjunath and Turhan (2016) satisfies the gradual safety property, in line with their Proposition 2.
} 
Definition 6 A rule $F \in \mathcal{F}$ is neutral if, for every $(P, v) \in \mathcal{P} \times(C \cup\{\emptyset\}), P^{\prime} \in F(P, v)$ implies $\sigma\left(P^{\prime}\right) \in F(\sigma(P), \sigma(v))$ for any permutation $\sigma: C \cup\{\emptyset\} \rightarrow C \cup\{\emptyset\} .{ }^{17}$

Definition 7 A rule $F \in \mathcal{F}$ satisfies the independence of irrelevant colleges if $c_{2} \in A_{P^{\prime}}$ for some $P^{\prime} \in F\left(P, c_{1}\right)$ implies $c_{2} \in A_{P^{\prime \prime}}$ for some $P^{\prime \prime} \in F\left(\hat{P}, c_{1}\right)$, for any $\hat{P}$ such that $c_{1} \hat{P} c_{2} \Leftrightarrow c_{1} P c_{2}$.

Proposition 4 Let $F \in \mathcal{F}$ be a refitting rule satisfying neutrality and independence of irrelevant colleges. Assume that $|C| \geq 3$. If $\mathcal{M}_{F}^{C O S M}$ is gradually safe then $F$ is strongly regular.

Proof See Appendix C.

\subsection{The case of single-valued refitting rules}

Single-valued refitting rules correspond to environments where ROLs are submitted once and for all in the first period. Once the ROL is submitted the sole degree of freedom left to the students is when to finalize their matchs. Note that single-valued refitting rules do not necessarily imply that the same ROL is used at each period. Single-valued refitting rules are of particular importance in practice. For instance, New York City recently announced that the two-round system used for middle and high school admissions will be replaced in 2020 by a one-round system with waiting list. Under this new system students will be put on a waiting list for any school that is ranked above their assignment in their submitted ROL. When seats become vacant (e.g., because of some students' withdrawals — see Section 6), waiting lists will be cleared using student's ROLs. It is not too difficult to see that this system is in fact akin to a gradual matching mechanism where the refitting rule is the truncation mapping, a single-valued refitting rule. ${ }^{18}$

In what follows we consider more specifically the identity mapping and the truncation mapping, as defined in Section 3.3. Recall that the identity mapping (resp. truncation

\footnotetext{
${ }^{17}$ As usual, $\sigma(P)$ is defined as $v \sigma(P) v^{\prime}$ if, and only if $\sigma(v) P \sigma\left(v^{\prime}\right)$.

${ }^{18}$ The procedure used in New York City does not perfectly match our model because schools' priority rankings may change slightly between rounds. Under some conditions students are allowed to be put in the waiting list for a school ranked below their proposal. Also, priority rankings may also be updated depending when taking into account the enhanced priority that low income students and students from minorities may benefit.
} 
mapping) is regular (resp. strongly regular). We start with a corollary of Theorem 1 and Proposition 2.

Corollary $2 \mathcal{M}_{F^{I D}}^{S O S M}$ and $\mathcal{M}_{F^{T R}}^{C O S M}$ are gradually safe.

To go further we observe that imposing one of those two rules implies that the relative order of acceptable colleges are unchanged in the sequences of ROLs. It allows us to make comparisons between the gradual matching obtained and the matching that would result from the static matching mechanism (i.e., the one obtained in the first period). It turns out that $\mathcal{M}_{F}^{S O S M}$ and $\mathcal{M}_{F}^{C O S M}$ perform very differently on that matter.

The next result establishes that gradual mechanisms have no raison d'être when the spot mechanism is COSM and the refitting rule is the truncation mapping. ${ }^{19}$ For every student, the first period assignment turns out to be the sole achievable one.

Proposition 5 For every problem $\boldsymbol{\Gamma}=\left(I^{1}, C,\left(P_{c}, q_{c}^{1}\right)_{c \in C}, \mathbf{P}_{I^{1}}\right)$, the gradual matching of $\mathcal{M}_{F^{T R}}^{C O S M}(\boldsymbol{\Gamma})$ is the matching $\operatorname{COSM}\left(I^{1}, C,\left(P_{c},\left(q_{c}^{1}\right)_{c \in C}\right),\left(P_{i}^{1}\right)_{i \in I^{1}}\right)$.

Proof See Appendix C.

A superficial reading of Proposition 5 would lead the reader to conclude that COSM is consistent, where consistency of a matching mechanism means that once we have removed from the problem a subset of agents (together with their match) the match of the remaining agents (with the same mechanism) should not change if we re-run the matching algorithm. The type of situations considered by the consistency property is thus similar to the problem studied here because under single-valued refitting rules a gradual matching mechanism simply implies of running over and over again the spot mechanism where some students may be withdrawn (together with their match) from the problem between two runs. Interpreting thus that Proposition 5 establishes the consistency of COSM would then be at odds with the well-known fact that the stability operator is not consistent (e.g., see Ergin, 2002). The key difference here is the presence of the refitting rule, which implies that the ROLs of students present in period 2 are not the same as the one they had in period $1 .^{20}$

We now turn to SOSM and recover an existing result of the literature. With an abuse of language we say that $\mathcal{M}_{F}^{S O S M}$ Pareto-dominates SOSM if the two matching mechanisms do not always produce the same final matchings and if, for any problem $\Gamma^{1}=$

\footnotetext{
${ }^{19}$ Unless some withdrawals occur, see Section 6.

${ }^{20}$ Example 2 shows that Proposition 5 does not hold if the refitting rule is the identity mapping.
} 
$\left(I^{1}, C,\left(P_{c}, q_{c}^{1}\right)_{c},\left(P_{i}^{1}\right)_{i \in I^{1}}\right)$ and any list of ROLs $\mathbf{P}_{I}$ such that $P_{i}^{t}=P_{i}^{1}$ and $2 \leq t \leq t_{i}$ for every $i \in I^{1}$, the gradual matching $\nu$ associated to the outcome $\mathcal{M}_{F}^{S O S M}\left(I^{1}, C,\left(P_{c}, q_{c}^{1}\right)_{c \in C}, \mathbf{P}_{I^{1}}\right)$ is such that, for every $i \in I^{1}, \nu R_{i}^{1} \operatorname{SOSM}\left(\Gamma^{1}\right){ }^{21}$

Though stated differently, it is an easy matter to verify that the next result is equivalent to Theorem 1 of Doğan and Yenmez (2018), which states that if students' only variable decision is when to finalize their match (i.e., at each period their submitted ROLs are their true preferences) then any gradual matching weakly Pareto dominates the student-optimal matching when the spot mechanism is SOSM. Here, it is obtained as a restatement of Corollary 2 by simply rephrasing the notion of gradual safety in terms of Pareto dominance, in this environment.

\section{Corollary $3 \mathcal{M}_{F^{I D}}^{S O S M}$ Pareto-dominates SOSM. ${ }^{22}$}

Proposition 5 shows that, without the ability to revise their ROLs, under COSM students do not gain by finalizing their match at later periods, for the final assignments are fully determined in first period. In contrast, Corollary 3 shows that under SOSM, even if ROLs are fixed, exiting at later periods may pay off.

Corollary 3 also permits us to make the link with Kesten (2010). Both gradual matching mechanisms and Kesten's EADAM mechanism consist of several iterations of a matching mechanism (SOSM in the case of EADAM) where student's ROLs are updated after each iteration. Recall that in EADAM a student's ROL is updated only if he is an interrupter and the school for which he is an interrupter is simply dropped from his ROL, leaving the relative ordering of the other schools unchanged. This updating would thus entail in a single-valued refitting rule if EADAM were framed as a gradual matching mechanism. However, further scrutiny shows that in fact EADAM substantially differs from gradual mechanisms. First, in EADAM the number of rounds (periods in our model) is not fixed, for it depends on the profile of ROLs. Second, in EADAM all students only finalize their match at the last period. Therefore, that students' decisions do no longer have an impact on the final outcome past the first period (i.e., there is only one possible execution path after the first period). Finally, EADAM as a gradual mechanism with a single-valued refitting rule does not entirely fit our model because the identity of an interrupter (and the corresponding school) does depend on

\footnotetext{
${ }^{21}$ Recall that we consider the profiles of ROLs as lists submitted by the students and not as their (genuine) preferences.

${ }^{22}$ Example 1 (scenario 1) shows that the final matchings of SOSM and $\mathcal{M}_{F^{I D}}^{S O S M}$ are not always identical.
} 
the complete profile of ROLs and priorities, and not on the interrupter's ROL only.

\section{Gradual stability}

So far we have only considered the problem of dynamic incentives that students may face in a system with gradual admissions; the question of stability was only a concern for the spot mechanism. In this section we explore the question of stability in gradual matching problems. Since students in a gradual outcome may not all be matched at the same period and, above all, since students ROLs may change from one period to the next, the concept of stability for static matching problems is obviously not suited for gradual outcomes, and not satisfied in general (see Example 1). We propose here a concept of gradual stability, an extension of the static stability concept to the case of gradual matching problems.

In static matching mechanisms stability considers only the final matching. For instance, the intermediate matchings that arise when running the Deferred Acceptance algorithm are not taken into account when checking stability. For gradual matchings this is not quite the case because of the mix centralized-decentralized nature of gradual matching mechanisms: the periods at which students finalize their matches are not decided by the mechanism. In other words, a student's match at any non-terminal can potentially be his final matching. Consequently, claims by a student (relative to justified envy or wastefulness) must be checked at all periods that student is active and not only with respect to his final match.

Our concept of gradual stability relies on the following principle: when a student finalizes his match in a gradual mechanism he exits the mechanism, thus waiving all the claims he could have about the matchings that could arise after he left. In other words, students' claims are legitimate only for the matchings that arose while the student was participating. That is, claims will necessarily be backward looking. However, non-contemporeanous claims will only be made with the realized matchings of the other students. That is, for $i$ and $j$ such that $t_{j}<t_{i}$, justified envy by $i$ against $j$ is checked for all periods $t_{j} \leq t \leq t_{i}$ using $i$ 's ROL and match at period $t$ and $j$ 's match at $t_{j}$ (and not $j$ 's match for some period $t^{\prime}<t_{j}$ ). For instance, if we consider the period $t$ just after $j$ finalized his match, i.e., $t=t_{j}+1$, justified envy takes the following form,

$$
\mu^{t_{j}}(j) P_{i}^{t} \mu^{t}(i) \quad \text { and } \quad i P_{\mu(j)} j
$$


If, however, $i$ remains active at $t+1$ the justified envy condition becomes

$$
\mu^{t_{j}}(j) P_{i}^{t+1} \mu^{t+1}(i) \quad \text { and } \quad i P_{\mu(j)} j
$$

The adaptation of the non-wastefulness criterion to our environment follows the same principle, i.e., at any period $t$ where $i$ is active (i.e., $t \leq t_{i}$ ) we check non-wastefulness with respect to all schools and all previous periods $t^{\prime} \leq t$ with respect to the school's capacity at period $t^{\prime}$.

Individual rationality takes a particular twist in gradual matching problems. In the standard, static case individual rationality is defined by comparing a matching with the empty matching, which is usually interpreted as some (unspecified) "outside option." This is fine for static problems that are taken in isolation: being not matched is the same as being matched with an "outsider." In a dynamic problem making this equivalence is a bit dicey because matches from previous periods do represent outside options. If, at any period, the current matching satisfies some notion of optimality, i.e., gradual stability, it must be that each student's match is ranked higher than his (past) outside options. Our concept of gradual stability is given by the following definition. ${ }^{23}$

Definition 8 An outcome $\left(\Gamma^{t}, \mu^{t}\right)_{t \leq T}$ is gradually stable if

(i) For each $i \in I^{1}, \mu^{t}(i) R_{i}^{t} \emptyset, \forall t \leq t_{i}$;

(ii) For each $i \in I^{1}, \mu^{t}(i) R_{i}^{t} \mu^{t^{\prime}}(i), \forall t^{\prime} \leq t \leq t_{i}$;

(iii) For each $i \in I^{1}$ and $c \in C, c P^{t} \mu^{t}(i)$ implies $\left|\mu^{t^{\prime}}(c)\right|=q_{c}^{t^{\prime}}, \forall t^{\prime} \leq t \leq t_{i}$;

(iv) For each $i, j \in I^{1}$ such that $t_{j} \leq t_{i}$ and $c \in C, \mu^{t_{j}}(j)=c$ and $c P_{i}^{t} \mu^{t}(i)$ imply $j P_{c} i, \forall t_{j} \leq$ $t \leq t_{i}$.

Let $\varphi$ and $F \in \mathcal{F}$ be respectively a spot mechanism and a refitting rule. The gradual matching mechanism $\mathcal{M}_{F}^{\varphi}$ is gradually stable if, for every $\boldsymbol{\Gamma}=\left(I^{1}, C,\left(P_{c}, q_{c}^{1}\right)_{c \in C}, \mathbf{P}_{I^{1}}\right)$, the outcome $\mathcal{M}_{F}^{\varphi}(\boldsymbol{\Gamma})=\left(\Gamma^{t}, \mu^{t}\right)_{t \leq T}$ is gradually stable.

Our notion can be related to the one used in the context of seniority based rules as in Pereyra (2013). Indeed, in teachers' assignment problems, a meaningful notion of stability

${ }^{23}$ Note that if there is only one period gradual stability is identical to the static stability defined in Section 2. 
does not usually consider justified claims over assignments made in past periods. In such environments, stability is merely based only on spot stability (period per period) and on a condition that guarantees the seniority rights, a requirement akin to gradual safety. The existence of stability is achieved through priorities, which are modified according to seniority rules, without assuming any restriction on the refitting rules (see the beginning of Section 4 for more details). This does not allow to keep track of backward justified claims, which is precisely the additional property we consider in Definition 8 (condition $(i v)){ }^{24}$

The next theorem is our second main result. It clarifies the new notion of gradual stability by establishing a direct connection with gradual safety, when it is associated to a stable spot matching mechanism.

Theorem 2 Let $F \in \mathcal{F}$ be a regular refitting rule. A gradual matching mechanism $\mathcal{M}_{F}^{\varphi}$ is gradually stable if, and only if, it is gradually safe and $\varphi$ is a stable spot mechanism.

Proof See Appendix C.

The next result is deduced from Proposition 2 and Theorems 1 and 2 .

\section{Corollary 4}

- If $F \in \mathcal{F}$ is strongly regular and $\varphi$ is a stable spot mechanism then $\mathcal{M}_{F}^{\varphi}$ is gradually stable.

- If $F \in \mathcal{F}$ is regular then $\mathcal{M}_{F}^{S O S M}$ is gradually stable.

- If $F \in \mathcal{F}$ is strongly regular then $\mathcal{M}_{F}^{C O S M}$ is gradually stable.

\section{Withdrawals}

In practice, it may happen that at a non-terminal period a student, who finalized his match at a previous period, frees the seat at the college he has been assigned to. From the perspective

\footnotetext{
${ }^{24}$ Different contexts may motivate reversed conceptual viewpoints. For instance, forward looking notions of stability appear in the contexts of a multi-time matching process or overlapping long-lived agents. Kennes et al. (2014) consider agents who, at any given date of their lifetimes, consume their "spot" matches and need also to evaluate what will be their matches in the future periods. In Doval (2019), in a model with irreversible matches, forward looking is inherent to the fact that the opportunities come from the arrival of new participants in the subsequent periods.
} 
of the initial problem that student ends up being unmatched. However, the seat that was occupied becomes then available for the students who are still active. Such situations can occur for instance when some students are involved simultaneously in separate competing matching mechanisms and leave some of them at some period or withdraw from the least preferred assignments. This is the type of situation analyzed by Manjunath and Turhan (2016), Doğan and Yenmez (2019) or Ekmekci and Yenmez (2019). ${ }^{25}$ Withdrawals can also occur when students have to pass an exam to get access to colleges but the gradual matching mechanism starts before the test. Withdrawals then happen when a student is assigned to a college but fails the entry exam. This is what happened for instance in France (see Section 7).

Remark 3 In static matching settings students' withdrawals are usually taken into account through a "scrambling" session, where unassigned seats and seats left vacant because of withdrawals are usually (but not always) offered to any participant who remained unmatched at the end of the main session. The two sessions together correspond to a gradual matching but does not fit our model. The key differences lies in the refitting rules and whether students participate to the matching mechanism of the next period. First, students who are assigned to a school in the main session are usually not allowed to participate to the scrambling session. Second, students' permitted ROLs in the scrambling session do not depend on their main session ROLs. That is, the refitting rule for those students is obviously not regular, and thus the mechanism is neither gradually safety nor gradually stable.

Withdrawals can be easily encoded into our modeling. The general structure is unchanged except that we now allow any student $i$ who finalized his match at period $t_{i}$, to notify a withdrawal at a later period, which we denote $\bar{t}_{i}$. When this happens the student remains inactive but his seat now becomes vacant. An illustration is provided in Example 1 (scenario $3)$.

Definition 9 A gradual outcome with withdrawals is given by $\left(\left(\Gamma^{t}, \mu^{t}\right)_{t \leq T},\left(\bar{t}_{i}\right)_{i \in I^{1}}\right)$ where:

\footnotetext{
${ }^{25}$ Manjunath and Turhan (2016) propose a multiple stage assignment process to deal with the vacant seats, where students withdraw from all the enrollment systems but one, at every period. As indicated in sub-Section 3.3, it follows that every system can be viewed as a gradual mechanism with the refitting rule $F^{M T}$. Doğan and Yenmez (2019) compare the performance of unified and divided enrollment systems in terms of efficiency. Ekmekci and Yenmez (2019) analyze the coexistence of multiple enrollment systems from the strategic viewpoint of the colleges.
} 
(a) for each $i \in I, \bar{t}_{i}=0$ (no withdrawal) or $\bar{t}_{i}>t_{i}$ (withdrawal at period $\bar{t}_{i}$ ),

(b) the sequence $\left(\Gamma^{t}, \mu^{t}\right)_{t \leq T}$ is feasible,

(c) for each period $1 \leq t<T$ and each college $c \in C$,

$$
\begin{aligned}
q_{c}^{t+1}= & \underbrace{\left(q_{c}^{t}-\left|\mu^{t}(c)\right|\right)}_{\text {\#vacant seats }} \\
+ & \underbrace{\left.\left|\left\{i \in I^{t+1}: \mu^{t}(i)=c\right\}\right|\right\}}_{\text {\#seats left by withdrawals }} \\
+ & \underbrace{\mid\left\{i \in I: \bar{t}_{i}=t+1 \text { and } \mu^{t_{i}}(i)=c\right\} \mid}_{\text {\#seats previously assigned to current active students }}
\end{aligned}
$$

The main novelty in Definition 9 is Eq. (9). At each period, a college's capacity is updated by first adding the vacant seats at the previous period and the number of students who were assigned to that college and have not yet finalized their match, and second by adding the number of students who were previously assigned to that college (and have finalized their matched earlier) but who just withdrew from the problem.

The sequence of submitted ROLs of every student $i$ is described by a pair $\widetilde{\mathbf{P}}_{i}=\left(\left(P_{i}^{t}\right)_{t \leq t_{i}}, \bar{t}_{i}\right)$. Given a spot mechanism $\varphi$ and a refitting rule $F$, the gradual matching mechanism is now denoted by $\mathcal{W} \mathcal{M}_{F}^{\varphi}$; it maps the sequence college admission problems and sequences of ROLs to gradual outcomes with withdrawals.

The key difference between a gradual matching mechanism and a gradual matching mechanism with withdrawals is how colleges' capacities evolve from one period to the next. However, our solution concepts being essentially backward looking, withdrawals do not jeopardize our previous results. The reason is twofold. First, a student's withdrawal makes no change for those who have accepted earlier a proposal. Second, for the remaining students, the withdrawal offers a new vacant seat that can be assigned if needed. Intuitively it should benefit the students. Hence gradual safety and gradual stability can be obtained under identical (strong) regularity conditions.

Proposition 6 Let $\varphi$ be a stable spot mechanism and let $F \in \mathcal{F}$ be a strongly regular refitting rule. Then $\mathcal{W} \mathcal{M}_{F}^{\varphi}$ is gradually safe and gradually stable.

Proposition 7 Let $F \in \mathcal{F}$ be a regular refitting rule. Then $\mathcal{W} \mathcal{M}_{F}^{S O S M}$ is gradually safe and gradually stable. 
The proof that $\mathcal{W} \mathcal{M}_{F}^{S O S M}$ is gradually safe is similar to that of Theorem 1 and is thus omitted. Similarly, the proof that $\mathcal{W} \mathcal{M}_{F}^{\varphi}$ is gradually safe for any strongly regular refitting rule and any stable spot mechanism is almost identical to that of Proposition 2. The unique difference in both cases relies on Equation (1). If the equation is replaced by Equation (9), i.e., the one describing the capacities of colleges in the gradual outcomes with withdrawals, it is an easy matter to check that all the arguments of the proofs go through. Gradual stability in Proposition 6 and Proposition 7 follows from a straightforward adaptation of the proof of Theorem 2, which is also omitted.

\section{The French college admissions system}

Until 2017, the French Ministry of Higher Education used a matching mechanism to assigning students to the higher education programs, "Admission Post-Bac" (APB), that had both centralized and decentralized parts. ${ }^{26,27}$

The APB mechanism consisted of three periods, running from early June to mid-July. At each period students were matched to a school through a college-proposing Deferred Acceptance algorithm, hence COSM, and were given the option to accept or decline their match (if any). ${ }^{28}$ Students declining their match could participate to the next period mechanism by submitting a new ROL over colleges.

Under APB withdrawals were substantial. The main reason is that, APB's first period was before students knew whether they passed the qualifying exam to enroll a higher education program (the baccalauréat) and usually around $20 \%$ of the students fail that exam. Another reason is that APB did not include all higher education programs. For instance, Université Paris-Dauphine, Sciences-Po, or nursery schools were not participating to APB. Hence, a non-negligible number of the students who participated to APB and got assigned to a program through APB eventually withdrew after having been admitted to a program

\footnotetext{
${ }^{26}$ The mechanism was open to all students in their last year of high school. In 2017, over 800,000 French high-school students used the platform.

${ }^{27} \mathrm{APB}$ is no longer in place in France and is now replaced by a sequential matching system akin to a decentralized implementation of the Deferred Acceptance algorithm (with colleges proposing).

${ }^{28} \mathrm{We}$ focus only on the multi-period aspect of the French system. Among other aspects, we disregard the details about the program's priority rankings over students. They contain large indifference classes where tie-breaking is depending among other things on the programs' rank in students' ROLs. See Bonkoungou (2017) for an in-depth analysis of different tie-breaking rules (although in a static setting).
} 
outside APB.

Students participating to the second and third round were constrained with respect to which new ROL they could submit. To identify precisely the properties of APB, we need first to identify the properties of the refitting rule used by APB. The rule was in fact relatively simple. For both the second and the third period, a new ROL was built from the ROL of the previous period by removing any program that was ranked below the proposal. In addition students were permitted to

(i) remove from the ROL of the previous period the proposal of the previous period;

(ii) remove from the ROL of the previous period some of programs that were ranked higher than the proposal;

A formal description is the following. ${ }^{29}$

Definition 10 The APB rule $F^{A P B}$ is such that, for each $(P, v) \in \mathcal{P} \times(C \cup\{\emptyset\})$ and $P^{\prime} \in F(P, v)$,

(i) For each $c, c^{\prime} \in A_{P^{\prime}}, c P^{\prime} c^{\prime} \Rightarrow c P c^{\prime}$.

(ii) For each $c$ such that $v P c, c \notin A_{P^{\prime}}$.

(iii) There is no refitting rule $F^{\prime}$ that satisfies (i) and (ii) and such that $F^{A P B}(P, v) \subset$ $F^{\prime}(P, v)$ for some $(P, v) \in \mathcal{P} \times(C \cup\{\emptyset\})$.

Hence, $F^{A P B}$ must satisfy a truncation property together with the fact that the relative ordering of colleges is unchanged among the remaining acceptable colleges. For instance, if $P=\left[c_{1}, c_{2}, c_{3}, c_{4}, \emptyset, \ldots\right]$ and $c_{3}$ is proposed at some stage the permitted ROLs are given by: $F^{A P B}\left(P, c_{3}\right)=\left\{\left[c_{1}, \emptyset, \ldots\right],\left[c_{2}, \emptyset, \ldots\right],\left[c_{3}, \emptyset, \ldots\right],\left[c_{1}, c_{2}, \emptyset, \ldots\right],\left[c_{1}, c_{3}, \emptyset, \ldots\right],\left[c_{2}, c_{3}, \emptyset, \ldots\right]\right.$, $\left.\left[c_{1}, c_{2}, c_{3}, \emptyset, \ldots\right],[\emptyset, \ldots]\right\}$. Clearly, the APB refitting rule is strongly regular. Applying Proposition 6 , we obtain thus the following result.

\footnotetext{
${ }^{29}$ In practice, students choose among those options at every round: YES: you accept definitely the proposal and you are removed from the pool of candidates; YES BUT (not available in the last round): you accept temporarily the proposal, but you wish to remain in the pool of candidates to obtain a college at least as good as the proposal (as long as the latter remains acceptable in your ROL); NO BUT (not available in the last round): you decline the proposal, which is removed definitely from your ROL list, but you wish to remain in the pool of candidates in the next periods, you might end up with no college; NO: you withdraw from APB. It can be shown that those options are encapsulated into the definition of the APB refitting rule.
} 
Proposition 8 The French college admissions system is gradually safe and gradually stable.

Our results suggest possible adjustments of the French system. First, the properties of gradual safety and gradual stability can be achieved by using different stable spot mechanisms. Instead of COSM, the use of SOSM also guarantees both properties. Second, the APB refitting rule is restrictive and can be relaxed, allowing students to submit among a larger set of ROLs without affecting the main properties of the system.

\section{Conclusion}

We analyze a new class of matching mechanisms. Their specificity relies on the conjunction of at least two original aspects. First, the one-time matching system allows the students to be matched at a chosen period of time. Second, students have the opportunity to update their ROLs at every period. Our main results identify the degree of freedom we can leave to students to maintain incentives to participate (gradual safety) and fairness (gradual stability), according to the submitted ROLs. Most remarkably, those restrictions are consistent with those observed on a real-life, large-scale market like the French college admissions system that compels the students to a strongly regular refitting rule.

Not surprisingly, those mechanisms are not immune against the misrepresentations of preferences. Even worse, bad incentives are more pervasive in this context and materialize into more dimensions (strategic decision timing and preference revelation across periods). Doğan and Yenmez (2018) have identified this limitation even in the specific case of gradual admissions with no refitting. Despite this issue, gradual matching mechanisms, in contrast to one-shot mechanisms, provide an adequate framework to account for withdrawals, to offer students the possibility to adjust their preferences or ROLs, or to allow students to express time preferences about the date at which they are matched. 


\section{A. Gradual safety: backward and forward looking}

A spot mechanism $\varphi$ is individually rational if, for every college admission problem $\Gamma$, the matching $\varphi(\Gamma)$ is individually rational.

Lemma 1 Let $\varphi$ be an individually rational spot mechanism and $F \in \mathcal{F}$ be regular. If an outcome $\left(\Gamma^{t}, \mu^{t}\right)_{t}$ is gradually safe then, for each $2 \leq t \leq T$ and $i \in I^{t}$,

$$
\mu^{t-1}(i) \in A_{P^{t}} \Rightarrow \mu^{t} R_{i}^{t-1} \mu^{t-1}
$$

Proof Suppose on the contrary that $\mu^{t-1} P_{i}^{t-1} \mu^{t}$ with $\mu^{t-1}(i) \in A_{P^{t}}$, it follows that $\mu^{t-1}(i) \neq \mu^{t}(i)$. Since $\varphi$ is individually rational, we know that $\mu^{t} R_{i}^{t} \emptyset$. If $\mu^{t}(i) \in C$ then, from regularity, it must be the case that $\mu^{t-1}(i) P_{i}^{t} \mu^{t}(i)$, which contradicts gradual safety (Eq. (5)). If $\mu^{t}(i)=\emptyset$, Eq. (5) implies that $\mu^{t-1} \notin A_{P^{t}}$, which is again a contradiction.

The condition $\mu^{t-1}(i) \in A_{P^{t}}$ in Eq. (10) is crucial and necessary. If it were not satisfied, that is, if $\mu^{t-1}(i)$ is not acceptable for the ROL $P^{t}$, one cannot guarantee that, at the end of each non-final period, students are incentivized to participate to the next period's matching, in the sense of Eq. (6). A trivial counter-example is the situation where a student gets a college at period $t-1$ and $P^{t}=[\emptyset, \cdots]$. Such a ROL is consistent with our regularity condition, but for any individually rational spot mechanism we then have $\mu^{t}(i)=\emptyset$, which violates Eq. (6).

A natural way to guarantee the condition $\mu^{t-1}(i) \in A_{P^{t}}$ is by modifying condition (i) we impose on every $F \in \mathcal{F}$.

$\left(i^{\prime}\right)$ For every $(P, v) \in \mathcal{P} \times(C \cup\{\emptyset\})$, if $v \in C$ then $v \in A_{P^{\prime}}$ for every $P^{\prime} \in F(P, v)$.

Denote by $\widetilde{\mathcal{F}}$ be the set of all refitting rules that satisfy conditions $\left(i^{\prime}\right)$ and $(i i)$. By using Lemma 1 and our former results we easily obtain the following corollaries.

Corollary 5 Let $\varphi$ be a stable spot mechanism and $F \in \widetilde{\mathcal{F}}$ be strongly regular. Then the mechanism $\mathcal{M}_{F}^{\varphi}$ satisfies Equation (6).

Corollary 6 Let $F \in \widetilde{\mathcal{F}}$ be regular. Then the mechanism $\mathcal{M}_{F}^{S O S M}$ satisfies Equation (6).

Finally, consider the case of the so-called scrambling markets, where only participants that are unmatched in the initial (main) period are allowed to participate in a second run of 
the matching mechanism. In such cases only colleges with unfilled capacities are available. Provided the spot mechanism is non-wasteful the second period match will be necessarily weakly better than the first period matching. That is, such mechanisms are trivially gradually safe. However, note that Eq. (6) is not satisfied.

\section{B. Strategic behavior in gradual matching mechanisms}

The strategic issues associated to the setting of gradual matching mechanisms are set aside in the paper. Doğan and Yenmez (2018) provides a thorough analysis of incentives in this environment. Before reviewing the main insights of their contribution, recall that, contrary to standard static mechanisms, strategy sets in a gradual matching mechanism do not boil down to submitting a ROL once and for all, for students may submit several ROLs and have also to decide at each non-terminal period whether to finalize their match. That is, the strategy sets are more complex and much larger than in the static case.

Doğan and Yenmez (2018) main focus is to compare students' welfare between gradual matching mechanism over two periods and the matching from a static mechanism. The spot mechanism used in the multi-period setting and the static mechanism are the same, namely SOSM. Another important difference is that they conduct an equilibrium analysis; they consider subgame-perfect Nash equilibria of the gradual mechanism.

Their first result, in line with our Corollary 3, shows that if the students are not strategic in their preferences (i.e., they submit their true preferences at every period), then the gradual mechanism Pareto dominates the static mechanism (with students submitting their true preferences, too). In addition, they show that strict Pareto improvement can be realized only if the priority profile of colleges admits a cycle à la Ergin, for otherwise SOSM is consistent and thus students' matches under SOSM are not affected by the early departure of other students (i.e., the outcomes are all the same for any profile of exit decision). In the case of fully strategic students, i.e., the students can report strategically their preferences in the first period, submit their true preferences in the second period and decide whether to move to the second period, they obtain two contrasted results. If the priority profile admits a cycle, there exists an outcome of the gradual mechanism that Pareto dominates the truthful equilibrium given by SOSM in the static case. If the priority profile is acyclic, then the truthful equilibrium given by SOSM in the static case is always weakly preferred to any outcome of the gradual mechanism. Hence, their welfare comparisons lead to sharp 
conclusions.

We want to emphasize that there is another complication related to strategies, which is rather implicit in the contribution of Doğan and Yenmez (2018). Gradual mechanisms are essentially not strategyproof even in the case where students can report only once their preferences during the multi-period procedure. The following example illustrates a manipulation from a student in the case where the spot mechanism is SOSM (and thus strategyproof for the students in a static mechanism). Interestingly, it does not rely on the possibility of refitting across periods but only on the exogenous differentiated acceptance dates. That sole detour from the static model is sufficient to give room for manipulation in the first period.

Example 3 We assume for simplicity that the students have time-independent preferences over colleges. That is, each student has a true ROL over colleges given once and for all at the beginning of the procedure. Note that here we deviate here from the model in the paper because we consider students' preferences and not their submitted ROLs.

Consider three colleges, $c_{1}, c_{2}, c_{3}$, and three students, $i_{1}, i_{2}, i_{3}$. Each college has one seat to offer. Let the students' true preferences and colleges' priorities be summarized by Table 3. Under SOSM the first period matching is $\left(i_{1}, \emptyset\right),\left(i_{2}, c_{2}\right),\left(i_{3}, c_{1}\right)$.

\begin{tabular}{ccccccc}
$P_{i_{1}}$ & $P_{i_{2}}$ & $P_{i_{3}}$ & & $P_{c_{1}}$ & $P_{c_{2}}$ & $P_{c_{3}}$ \\
\cline { 1 - 2 }$c_{1}$ & $c_{2}$ & $c_{1}$ & & $i_{2}$ & $i_{1}$ & $i_{3}$ \\
& $c_{1}$ & $c_{2}$ & & $i_{3}$ & $i_{3}$ & \\
& & $c_{3}$ & & $i_{1}$ & $i_{2}$ &
\end{tabular}

Table 3: Simple manipulation, without refitting

Suppose that $i_{3}$ 's strategy consists of leaving at the end of period 1 , while $i_{1}$ and $i_{2}$ plan to remain until the end of period 2 , and consider the following strategy for $i_{1}$ : submitting $P_{i_{1}}^{\prime}=\left[c_{1}, c_{2}, \emptyset, \ldots\right]$ (and still planning to participate in period 2's mechanism). In the first period, the computation of SOSM gives $\mu^{\prime}=\left(\left(i_{1}, c_{2}\right),\left(i_{2}, c_{1}\right),\left(i_{3}, c_{3}\right)\right)$. In the second period, $i_{1}$ obtains the seat in $c_{1}$ and $i_{2}$ obtains the seat in $c_{2}$ (recall that $i_{3}$ is gone). Hence, by using $P_{i_{1}}^{\prime}$ during the two periods she gets her first choice instead of remaining alone in the case of sincere submitted preferences. The intuition is straightforward. Manipulating cannot make the student $i_{1}$ better off in the first period (from the strategyproofness of SOSM). However by doing so student $i_{1}$ modifies the assignments of other students at period 1 , which can be 
beneficial in the next period.

Observe that the example entirely relies on the fact that the participants do not leave the market at the same period. It is a necessary condition in general to make a deviation profitable in gradual matching problems where the spot mechanism is SOSM and the refitting rule is the identity rule. Suppose on the contrary that the termination periods are all equal. Under the identity rule, the outcome of the mechanism must be such that the matchings generated at every period are all equal to the one of period 1 . Thus there is no profitable deviation for the students in the gradual matching mechanism since SOSM is strategyproof for the students.

\section{Proofs}

Proof of Proposition 1 Suppose by way of contradiction that $F$ is not regular, that is, there exist $\left(P, c_{1}\right) \in \mathcal{P} \times(C \cup\{\emptyset\})$ and a college $c_{2} \in A_{P}$ for some $P^{\prime} \in F\left(P, c_{1}\right)$ such that $c_{1} P c_{2}$ and $c_{2} P^{\prime} c_{1}$ with $c_{2} \in A_{P^{\prime}}$. Note that $c_{1}, c_{2} \in A_{P}$ by condition (ii) of a refitting rule $F \in \mathcal{F}$.

Let $\Gamma^{1}=\left(I^{1}, C,\left(P_{c}, q_{c}^{1}\right)_{c \in C},\left(P_{i}^{1}\right)_{i \in I^{1}}\right)$ be a (first period) college admission problem with $I^{1}=\left\{i_{1}, i_{2}, \ldots, i_{n}\right\}$ and $C=\left\{c_{1}, c_{2}, \ldots, c_{m}\right\}$, where $P_{i_{1}}^{1}=P, P_{i_{2}}^{1}=\left[c_{2}, \emptyset, \ldots\right], P_{c_{1}}=$ $\left[i_{1}, i_{2}, \ldots\right], P_{c_{2}}=\left[i_{1}, i_{2}, \ldots\right]$, and for each $c \neq c_{1}, c_{2}$, student $i_{1}$ has the lowest priority in $P_{c}$, and for each $i \neq i_{1}, i_{2}$, every college except $c_{1}$ and $c_{2}$ is acceptable at $P_{i}^{1}$. For each $c \in C$, $q_{c}^{1}=1$, and let $I^{1}$ be such that $\left|I^{1}\right| \geq|C|$.

Since $\varphi$ is a stable mechanism, $\mu^{1}:=\varphi\left(\Gamma^{1}\right)$ is necessarily such that $\mu^{1}\left(i_{1}\right)=c_{1}$ and $\mu^{1}\left(i_{2}\right)=c_{2}$. Let $\Gamma^{2}=\left(I^{2}, C,\left(P_{c}, q_{c}^{2}\right)_{c \in C},\left(P_{i}^{2}\right)_{i \in I^{2}}\right)$ be the second period problem such that $I^{2}=\left\{i_{1}, i_{2}\right\}$ (i.e., all the other students finalize their match in period 1 ). Note that $q_{c}^{2}=0$ for each $c \neq c_{1}, c_{2}$, by construction. Conditions $(i)$ and (ii) of a refitting rule $F \in \mathcal{F}$ imply that a permitted ROL for student $i_{2}$ is $P_{i_{2}}^{2}=\left[c_{2}, \emptyset, \ldots\right] \in F\left(P_{2}^{1}, c_{2}\right)$. The ROL $P_{i_{1}}^{2}=P^{\prime}$ is permitted for student $i_{1}$ since $P^{\prime} \in F\left(P, c_{1}\right)$. Since $c_{2} P^{\prime} c_{1}$, this yields under $\varphi$ the matching $\mu^{2}$ where $\mu^{2}\left(i_{1}\right)=c_{2}$ and thus $\mu^{2}\left(i_{2}\right)=\emptyset$. Therefore, $\mu^{1}\left(i_{2}\right) P_{i_{2}}^{2} \mu^{2}\left(i_{2}\right)$, and thus $\mathcal{M}_{F}^{\varphi}$ is not gradually safe, the desired result.

Proof of Proposition $2 \quad$ Consider the problem $\boldsymbol{\Gamma}=\left(I^{1}, C,\left(P_{c}, q_{c}^{1}\right)_{c \in C}, \mathbf{P}_{I^{1}}\right)$. Let $\left(\Gamma^{t}, \mu^{t}\right)_{t \leq T}$ be the outcome of $\mathcal{M}_{F}^{\varphi}$ for that problem. Suppose by way of contradiction that $\mu^{t-1}(i) P_{i}^{t} \mu^{t}(i)$ for some $t>1$ and $i \in I^{t}$. Since $\varphi$ is a stable spot mechanism, it holds that $\mu^{t}$ is stable 
for the problem $\Gamma^{t}$ at every period. From the individual rationality of $\mu^{t}$ for $\Gamma^{t}$ we have $\mu^{t}(i) R_{i}^{t} \emptyset$, hence $\mu^{t-1}(i)$ is necessarily a college. Let $c$ be that college.

From the non-wastefulness of $\mu^{t}$ for $\Gamma^{t}, c P_{i}^{t} \mu^{t}(i)$ implies that $\left|\mu^{t}(c)\right|=q_{c}^{t}$. From Equation (1), $q_{c}^{t} \geq\left|\left\{i \in I^{t}: \mu^{t-1}(i)=c\right\}\right|$. That is, the number of students assigned to $c$ at period $t$ is greater or equal to the number of seats of the college $c$ assigned at period $t-1$ to students still active at period $t$. Since $\mu^{t}(i) \neq \mu^{t-1}(i)=c$, there exists a student $j \in I^{t} \backslash\{i\}$ such that $\mu^{t}(j)=c$ and $\mu^{t-1}(j) \neq c$.

From the no justified envy condition, it holds that $j P_{c} i$ since $c P_{i}^{t} \mu^{t}(i)$. If $\mu^{t-1}(j) P_{j}^{t-1} c$ then $\emptyset P_{j}^{t} c$ under the strong regularity of $F$, but this contradicts that $\mu^{t}$ is individually rational for $\Gamma^{t}$. Since $\mu^{t-1}(j) \neq c$, it follows necessarily that $c P_{j}^{t-1} \mu^{t-1}(j)$. But $\mu^{t-1}(i)=c$ and $j P_{c} i$ imply together that $\mu^{t-1}$ does not satisfy the no justified envy condition for $\Gamma^{t-1}$, a contradiction.

Proof of Theorem 1 (if part) Let $\left(I^{1}, C,\left(P_{c}, q_{c}^{1}\right)_{c \in C}, \mathbf{P}_{I^{1}}\right)$ be the input of $\mathcal{M}_{F}^{S O S M}$ and consider the resulting outcome $\left(\Gamma^{t}, \mu^{t}\right)_{t \leq T}$. Let $1<t \leq T$. For each college $c$, define the sets $U_{c}$ and $V_{c}$ as follows:

$$
\begin{aligned}
& U_{c}=\left\{i \in I^{t}: \mu^{t}(i)=c \text { and } \mu^{t-1}(i) P_{i}^{t} c\right\}, \\
& V_{c}=\left\{i \in I^{t}: \mu^{t-1}(i)=c \text { and } c P_{i}^{t} \mu^{t}(i)\right\} .
\end{aligned}
$$

Claim $\left|U_{c}\right|=\left|V_{c}\right|, \quad \forall c \in C$.

Proof of the claim We first show that $\left|U_{c}\right| \geq\left|V_{c}\right|$ for each $c \in C$. To this end, suppose by way of contradiction that for some college $c$ we have $\left|V_{c}\right|>\left|U_{c}\right|$. So $V_{c} \neq \emptyset$, and thus there exists a student $j_{0} \in V_{c}$. Note that this does not necessarily mean that $j_{0} \in U_{c^{\prime}}$ for some college $c^{\prime}$ (if $\mu^{t}\left(j_{0}\right)=\emptyset$ then there is no such college $c^{\prime}$ ).

Define the following sets,

$$
\begin{aligned}
& A_{1}=\left\{i \in I^{t}: \mu^{t-1}(i)=\mu^{t}(i)=c\right\} \\
& A_{2}=\left\{i \in I^{t}: \mu^{t}(i) P_{i}^{t} \mu^{t-1}(i)=c\right\}
\end{aligned}
$$

From Equation (1) we know that:

$$
q_{c}^{t}=\underbrace{\left|A_{1}\right|+\left|A_{2}\right|+\left|V_{c}\right|}_{\text {\# seats assigned at } t-1 \text { to active stud. }}+\underbrace{q_{c}^{t-1}-\left|\mu^{t-1}(c)\right|}_{\text {\# vacant seats }}
$$


Define the following set,

$$
A_{3}=\left\{i \in I^{t}: c=\mu^{t}(i) P_{i}^{t} \mu^{t-1}(i)\right\} .
$$

Observe that $\mu^{t}(c)=A_{1} \cup A_{2} \cup U_{c}$ and that the three sets are mutually disjoint. It follows that

$$
\left|\mu^{t}(c)\right|=\left|A_{1}\right|+\left|A_{3}\right|+\left|U_{c}\right|
$$

Since $\mu^{t}$ is stable for $\Gamma^{t}, \mu^{t}$ is non-wasteful. It follows thus that $\left|\mu^{t}(c)\right|=q_{c}^{t}$ since $j_{0} \in V_{c}$. From Equations (13) and (14), it holds that

$$
\left|A_{3}\right|+\left|U_{c}\right|=\left|A_{2}\right|+\left|V_{c}\right|+q_{c}^{t-1}-\left|\mu^{t-1}(c)\right| .
$$

By construction, $q_{c}^{t-1}-\left|\mu^{t-1}(c)\right| \geq 0$. Hence, if $\left|V_{c}\right|>\left|U_{c}\right|$ then we must have $\left|A_{3}\right|>0$. Let $k \in A_{3}$. Since $\mu^{t}$ is stable for $\Gamma^{t}$ and $c P_{j_{0}}^{t} \mu^{t}\left(j_{0}\right)$, it must hold that $k P_{c} j_{0}$ (recall that $j_{0} \in V_{c}$ ). Since $c$ is a college and $c \in A_{P_{k}^{t}}$, it must hold that $c P_{k}^{t-1} \mu^{t-1}(k)$ because $F$ is regular. Since $\mu^{t-1}\left(j_{0}\right)=c$, this implies in turn that $\mu^{t-1}$ is not stable for $\Gamma^{t-1}$, a contradiction. So $\left|U_{c}\right| \geq\left|V_{c}\right|$ for each $c \in C$.

Since SOSM is a stable mechanism, $\mu^{t}$ is individually rational for $\Gamma^{t}$. Thus we have $\mu^{t-1}(j) \in C$ for each $j \in U_{c}$. So $j \in U_{c}$ implies $j \in V_{\mu^{t-1}(j)}$. Therefore, $\cup_{c} U_{c} \subseteq \cup_{c} V_{c}$. Since $\left|U_{c}\right| \geq\left|V_{c}\right|$ for each $c \in C$, we have $\left|U_{c}\right|=\left|V_{c}\right|$ for each $c \in C$ as was to be proved.

Suppose the conclusion of the proposition is not true at period $t$ for the outcome $\left(\Gamma^{t}, \mu^{t}\right)_{t \leq T}$. So $U_{c} \neq \emptyset$ for some $c$. Let $\widetilde{I}=\cup_{c} U_{c}$, and let $\widetilde{\mu}$ be the matching such that

- for each $i \in \widetilde{I}, \widetilde{\mu}(i)=\mu^{t-1}(i)$,

- for each $i \notin \widetilde{I}, \widetilde{\mu}(i)=\mu^{t}(i)$.

Note that for each $c, \widetilde{\mu}(c)$ is obtained by "replacing" the $U_{c}$ students by $V_{c}$ students. Since $\left|V_{c}\right|=\left|U_{c}\right|$, college $c$ is matched to $|\widetilde{\mu}(c)|=\left|\mu^{t}(c)\right|$ different students under $\widetilde{\mu}$. As for the students, observe that, for each $i \in \cup_{c} U_{c}$, there exists only one college $c^{\prime}$ such that $i \in U_{c^{\prime}}$ and only one college $c^{\prime \prime}$ such that $i \in V_{c^{\prime \prime}}$. So, each student $i \in I^{t}$ is matched to at most one college under $\widetilde{\mu}$, therefore $\widetilde{\mu}$ is a matching for $\Gamma^{t}$.

We claim that $\widetilde{\mu}$ is stable for $\Gamma^{t}$. To see this, suppose that there exist $(i, c)$ so that $c P_{i}^{t} \widetilde{\mu}(i)$ and $j \in \widetilde{\mu}(c)$ such that $i P_{c} j$. If $\widetilde{\mu}(c)=\mu^{t}(c)$, that is, $\mu^{t}(c) \cap \widetilde{I}=\emptyset$, then $(i, c)$ is such that $c P_{i}^{t} \mu^{t}(i)$ and $\mu^{t}$ is not stable for $\Gamma^{t}$, a contradiction. So, we can assume that 
$j \in \widetilde{I}$. Suppose first that $i \in \widetilde{I}$. Note that $\widetilde{\mu}(i)=\mu^{t-1}(i)$, so the application of the regularity assumption implies that $c P_{i}^{t-1} \mu^{t-1}(i)$. Since $j \in \widetilde{I}$, we have $\widetilde{\mu}(j)=\mu^{t-1}(j)$. So $i$ has justified envy against $j$ at period $t-1$, i.e., $\mu^{t-1}$ is not stable for $\Gamma^{t-1}$, a contradiction. Hence $i \notin \widetilde{I}$. So $\mu^{t}(i) R_{i}^{t} \mu^{t-1}(i)$, for otherwise we would have $i \in V_{\mu^{t-1}(i)}$ and thus $i \in \widetilde{I}$. It follows that $c P_{i}^{t} \mu^{t-1}(i)$. Using again the fact that $F$ is regular, we obtain $c P_{i}^{t-1} \mu^{t-1}(i)$. Since $\widetilde{\mu}(j)=\mu^{t-1}(j)$, student $i$ has thus a justified envy against $j$, which contradicts again the stability of $\mu^{t-1}$ for the problem $\Gamma^{t-1}$.

So at period $t$ we obtain two stable matchings, $\widetilde{\mu}$ and $\mu^{t}$. By construction, for each student $i \in \widetilde{I}, \widetilde{\mu} P_{i}^{t} \mu^{t}$, and $\mu^{t}(i)=\widetilde{\mu}(i)$ if $i \notin \widetilde{I}$. So $\mu^{t}$ cannot be the student-optimal student matching of $\Gamma^{t}$, a contradiction. So, $U_{c}=\emptyset$ for each $c \in C$, which completes the proof.

Proof of Proposition 4 Suppose by way of contradiction that $F$ is not strongly regular, that is, for some set of colleges $C$ there exist $\left(P, c_{1}\right) \in \mathcal{P} \times C$ and $c_{2} \in C$ such that $c_{1} P c_{2}$ and $c_{2} \in A_{\widetilde{P}}$ for some $\widetilde{P} \in F\left(P, c_{1}\right)$. Note that we do not know how $P$ ranks the other schools, that is, we only know that $c_{1}$ is ranked above $c_{2}$. We claim that we can assume without loss of generality that there exists $c_{3} \neq c_{1}, c_{2}$ such that $c_{1} P c_{3} P c_{2}$ and there is no college $c$ such that $c_{1} P c P c_{3}$ (i.e., $c_{3}$ is ranked just below $c_{1}$ ). To see this, note first that since $|C| \geq 3$, such a college $c_{3}$ exists. If $c_{1} P c_{3} P c_{2}$ with $c_{3}$ just below $c_{1}$ does not hold then consider the ROL $\breve{P}$ where $c_{1} \breve{P} c_{3} \breve{P} c_{2}$ (and $c_{3}$ just below $c_{1}$ ). By the independence or irrelevant colleges, $c_{2} \in A_{\widetilde{P}}$ for some $\widetilde{P} \in F\left(P, c_{1}\right)$ implies $c_{2} \in A_{\widetilde{P}}$ for some $\widetilde{P} \in F\left(\breve{P}, c_{1}\right)$, i.e., $F$ is not strongly regular for the ROL $\breve{P}$ and proposal $c_{1}$.

Case 1: $c_{2} \widetilde{P} c_{1}$ with $c_{2} \in A_{\widetilde{P}}$. This corresponds to the case where $F$ is actually not even regular. The proof for that case is identical to that of Proposition 1.

Case 2: $c_{1} \widetilde{P} c_{2} \widetilde{P} \emptyset . \quad$ Assume that, for each $c \in C, q_{c}^{1}=1$. Let $I^{1}$ be such that $\left|I^{1}\right|=|C|$ and let $c_{3} \neq c_{1}, c_{2}$. Since $\left|I^{1}\right|=|C| \geq 3$, there exists $i_{3} \neq i_{1}, i_{2}$. We follow a construction due to Segal (2007) to define the profile $\left(P_{I^{1}}^{1}, P_{C}\right)$ as follows. Let $\bar{\Gamma}=\left(\bar{I}, \bar{C},\left(\bar{P}_{c}, \bar{q}_{c}\right)_{c \in \bar{C}},\left(\bar{P}_{i}\right)_{i \in \bar{I}}\right)$ be an auxiliary college admission problem where $\bar{I}=I^{1} \backslash\left\{i_{3}\right\}, \bar{C}=C \backslash\left\{c_{3}\right\}, \bar{q}_{c}=1$ for every $c \in \bar{C}$, and $\left(\left(\bar{P}_{i}\right)_{i \in \bar{I}},\left(\bar{P}_{c}\right)_{c \in \bar{C}}\right)$ is any profile such that $\bar{P}_{i_{1}}$ is obtained from $P$ by dropping $c_{3}$, and $\bar{P}_{i_{2}}$ is the ROL obtained from $P$ by swapping $c_{1}$ and $c_{2}$ and dropping $c_{3}$ (i.e., $\bar{P}_{i_{2}}$ is $\bar{P}_{i_{1}}$ 
where $c_{1}$ and $c_{2}$ are swapped). The profile $\left(\bar{P}_{i}\right)_{i \in \bar{I} \backslash\left\{i_{1}, i_{2}\right\}}$ is any profile such that

$$
\begin{aligned}
\operatorname{SOSM}(\bar{\Gamma})\left(i_{1}\right) & =\operatorname{COSM}(\bar{\Gamma})\left(i_{2}\right)=c_{1}, \\
\operatorname{COSM}(\bar{\Gamma})\left(i_{1}\right) & =\operatorname{SOSM}(\bar{\Gamma})\left(i_{2}\right)=c_{2}, \\
\operatorname{SOSM}(\bar{\Gamma})(i) & =\operatorname{COSM}(\bar{\Gamma})(i) \quad \text { for } i \neq i_{1}, i_{2} \cdot{ }^{30}
\end{aligned}
$$

Note that since $c_{1} \bar{P}_{i_{1}} c_{2}$ and $c_{2} \bar{P}_{i_{2}} c_{1}$, the above outcomes imply $i_{2} \bar{P}_{c_{1}} i_{1}$ and $i_{1} \bar{P}_{c_{2}} i_{2}$.

Let $\mu^{1}$ be a matching for $I^{1}$ and $C$ defined by $\mu^{1}\left(i_{3}\right)=c_{3}$ and $\mu^{1}(i)=\operatorname{SOSM}(\bar{\Gamma})(i)$ for every $i \neq i_{3}$. By Lemma 5 of Segal (2007) there exists $\left(P^{1}{ }_{I^{1}}, P_{C}\right)$ such that $\mu^{1}$ is the unique stable matching for $\Gamma^{1}=\left(I^{1}, C,\left(P_{c}, q_{c}^{1}\right)_{c \in C},\left(P_{i}^{1}\right)_{i \in I^{1}}\right)$ and such that $\bar{P}_{\bar{I}}\left(\operatorname{resp} . \bar{P}_{\bar{C}}\right)$ is obtained from $P_{I^{1}}^{1}$ (resp. $P_{C}$ ) by simply dropping $c_{3}$ (resp. $\left.i_{3}\right)$ from the $\operatorname{ROLs}\left(P_{i}^{1}\right)_{i \in \bar{I}}$ (resp. priorities $\left.\left(P_{c}\right)_{c \in \bar{C}}\right)$. Note that Segal's construction requires that $c_{3}$ is ranked just below $c_{1}$ in $P_{i_{1}}^{1}$. Hence, $P_{i_{1}}^{1}=P$. Note also that $c_{3}$ is ranked just below $c_{2}$ in $P_{i_{2}}^{1}$ (which follows from our construction of $\bar{P}_{i_{2}}$ ). It follows that $P_{i_{2}}^{1}$ is simply obtained from $P_{i_{1}}^{1}$ by swapping $c_{1}$ and $c_{2}$.

Let $\Gamma^{2}=\left(I^{2}, C,\left(P_{c}, q_{c}^{2}\right)_{c \in C},\left(P_{i}^{2}\right)_{i \in I^{2}}\right)$ be the second period problem such that $I^{2}=\left\{i_{1}, i_{2}\right\}$ (i.e., all the other students finalize their match in period 1). Note that $q_{c}^{2}=0$ for each $c \neq c_{1}, c_{2}$. Let $P_{i_{1}}^{2}=\widetilde{P}$. So, $c_{1} P_{i_{1}}^{2} c_{2}$. Since $P_{i_{2}}^{1}$ is obtained from $P_{i_{1}}^{1}=P$ by swapping $c_{1}$ and $c_{2}$ there exists, by neutrality, $P^{\prime \prime} \in F\left(P_{i_{2}}^{1}, c_{2}\right)$ such that $c_{1} \in A_{P^{\prime \prime}}$. So, let $P_{i_{2}}^{2}=P^{\prime \prime}$. Under COSM we then have $\mu^{2}\left(i_{1}\right)=c_{2}$ and $\mu^{2}\left(i_{2}\right)=c_{1}$. We then have constructed a gradual outcome $\left(\left(\Gamma^{1}, \mu^{1}\right),\left(\Gamma^{2}, \mu^{2}\right)\right)$ for the mechanism $\mathcal{M}_{F}^{\varphi}$. However, since $\mu^{1} P_{i_{1}}^{2} \mu^{2}$ we obtain that $\mathcal{M}_{F}^{\varphi}$ is not gradually safe, the desired result.

Proof of Proposition 5 Consider the problem $\boldsymbol{\Gamma}=\left(I^{1}, C,\left(P_{c}, q_{c}^{1}\right)_{c \in C}, \mathbf{P}_{I^{1}}\right)$ and its outcome $\left(\Gamma^{t}, \mu^{t}\right)_{t \leq T}$. By assumption, for every student $i$ and all $2 \leq t \leq t_{i}, P_{i}^{t}$ is truncation of $P_{i}^{t-1}$ just below the proposal $\mu^{t-1}(i)$. To prove the proposition, it suffices to show that $\mu^{t}(i)=\mu^{1}(i)$ holds for each $t>1$ and $i \in I^{t}$, where $\mu^{1}$ is the COSM of $\Gamma^{t}$. The property is obviously true for $t=1$.

Consider the second period and denote by $\mu_{I^{2}}^{1}$ the matching $\mu^{1}$ restricted to the set of students $I^{2}$. From the definition of an outcome, $\mu_{I^{2}}^{1}$ is a well-defined matching for $\Gamma^{2}$. We claim that $\mu_{I^{2}}^{1}$ is a stable matching for the problem $\Gamma^{2}$. To see this, we observe that for each $i \in I^{2}, \mu^{1}(i) R_{i}^{2} \emptyset$ since $P_{i}^{2}$ is the truncation of $P_{i}^{1}$ below $\mu^{1}(i)$. That is, $\mu_{I^{2}}^{1}$ is individually rational for $\Gamma^{2}$. Let $i \in I^{2}$ and $c \in C$ be such that $c P_{i}^{2} \mu^{1}(i)$. Hence, $c P_{i}^{1} \mu^{1}(i)$, which implies

\footnotetext{
${ }^{30}$ It suffices for example to have $c_{h}$ being ranked top in $\bar{P}_{i_{h}}$ and student $i_{h}$ ranked top by $c_{h}$, for $h \geq 4$.
} 
$\left|\mu^{1}(c)\right|=q_{c}^{1}$ because $\mu^{1}$ being a stable matching for $\Gamma^{1}$. By construction, $\Gamma^{2}$ is such that $q_{c}^{2}=q_{c}^{1}-\left(\left|\mu^{1}(c)\right|-\mid\left\{i: t_{i}>1\right.\right.$ and $\left.\left.\mu^{1}(i)=c\right\} \mid\right)$. Since $\mu_{I^{2}}^{1}(c)=\left\{i: t_{i}>1\right.$ and $\left.\mu^{1}(i)=c\right\}$, $q_{c}^{2}=\left|\mu_{I^{2}}^{1}(c)\right|$, i.e., the matching $\mu_{I^{2}}^{1}$ is non-wasteful. Finally, let $i, j \in I^{2}$ with $\mu^{1}(j)=c \in C$ and suppose that $c P_{i}^{2} \mu^{1}(i)$. Since $F$ is the truncation mapping and $\mu_{I^{2}}^{1}$ is individually rational for $\Gamma^{2}$, we have $c P_{i}^{1} \mu^{1}(i)$. Since $\mu^{1}$ is stable for $\Gamma^{1}$, it holds that $j P_{c} i$. This implies in turn that $\mu_{I^{2}}^{1}$ satisfies also the no justified envy condition in the problem $\Gamma^{2}$. So $\mu_{I^{2}}^{1}$ is a stable matching for $\Gamma^{2}$, which proves the claim.

Consider $\mu^{2}$ (that is, $\operatorname{COSM}\left(\Gamma^{2}\right)$ ) and any student $i \in I^{2}$. If $\mu^{1}(i) \in C$, then since $P_{i}^{2}$ is the truncation of $P_{i}^{1}$ just below $\mu^{1}(i)$, there is no college $c$ such that $\mu^{1}(i) P_{i}^{2} c P_{i}^{2} \emptyset$, i.e., $\mu^{1}(i)$ is the least preferred acceptable college in $P_{i}^{2}$. Since $\mu_{I^{2}}^{1}$ is stable for $\Gamma^{2}$ and $\mu^{1}(i) \neq \emptyset$, by the Rural Hospital Theorem (Roth, 1986) there is no stable matching $\widehat{\mu}$ for $\Gamma^{2}$ such that $\widehat{\mu}(i)=\emptyset$. We can conclude that $\mu^{1}$ is $i$ 's least preferred match in a stable matching for $\Gamma^{2}$. If $\mu^{1}(i)=\emptyset$ then $\mu^{1}$ is also necessarily $i$ 's least preferred match in a stable matching for $\Gamma^{2}$ since any stable matching is individually rational. Hence, for each student $i \in I^{2}$, $\mu^{2}(i)=\mu^{1}(i)$. It follows that $\operatorname{COSM}\left(\Gamma^{2}\right)=\mu^{2}=\mu_{I^{2}}^{1}$ (recall that $\operatorname{COSM}\left(\Gamma^{2}\right)$ is the pessimal stable matching for the students in the problem $\Gamma^{2}$ ). The same arguments can be used for the subsequent periods.

Lemma 2 Let $\left(\Gamma^{t}, \mu^{t}\right)_{t \leq T}$ be a gradually safe outcome of $\mathcal{M}_{F}^{\varphi}$, where $\varphi$ is a stable spot mechanism and $F$ is a regular refitting rule. Let $i \in I^{1}$ and $t^{\prime}$ be such that, for some $t^{\prime} \leq t_{i}$, $c P_{i}^{t^{\prime}} \mu^{t^{\prime}}(i)$. Then $c P_{i}^{t} \mu^{t}(i)$ for all $t<t^{\prime}$.

Proof Suppose that $t^{\prime} \geq 2$ (otherwise there is nothing to show). Since $\mathcal{M}^{\varphi}$ is gradually safe, it holds that $\mu^{t^{\prime}}(i) R_{i}^{t^{\prime}} \mu^{t^{\prime}-1}(i)$. Thus, $c P_{i}^{t^{\prime}} \mu^{t^{\prime}}(i)$ implies $c P_{i}^{t^{\prime}} \mu^{t^{\prime}-1}(i)$ and $c P_{i}^{t^{\prime}} \emptyset$. Since $P_{i}^{t^{\prime}} \in F\left(P_{i}^{t^{\prime}-1}, \mu^{t^{\prime}-1}(i)\right)$ and $F$ is regular, we must have $c P_{i}^{t^{\prime}-1} \mu^{t^{\prime}-1}(i)$. From gradual safety, we have $\mu^{t^{\prime}-1}(i) R_{i}^{t^{\prime}-1} \mu^{t^{\prime}-2}(i)$, which implies that $c P_{i}^{t^{\prime}-1} \mu^{t^{\prime}-2}(i)$ and $c P_{i}^{t^{\prime}-1} \emptyset$. Since $P_{i}^{t^{\prime}-1} \in$ $F\left(P_{i}^{t^{\prime}-2}, \mu^{t^{\prime}-2}(i)\right)$ we must have $c P_{i}^{t^{\prime}-2} \mu^{t^{\prime}-2}(i)$. Continuing this way we eventually obtain $c P_{i}^{t} \mu^{t}(i)$ for all $t<t^{\prime}$.

Proof of Theorem 2 Observe that gradual safety follows directly from condition (ii) in Definition 8. We first show that if $\mathcal{M}_{F}^{\varphi}$ is gradually stable then $\varphi$ is a spot stable mechanism. To this end, let $\left(\Gamma^{t}, \mu^{t}\right)_{t \leq T}$ be a gradually stable outcome. Without loss of generality, assume that $I^{T} \neq \varnothing$, i.e., some students participate in the spot mechanism until the last period. 
Note that the conditions (i), (iii), and (iv) imply that $\mu^{T}$ is a stable matching for $\Gamma^{T}$. Consider now the outcome $\left(\bar{\Gamma}^{t}, \overline{\mu^{t}}\right)_{t \leq T}$ defined as follows,

$$
\begin{array}{ll}
\bar{\Gamma}^{t}=\Gamma^{t} & \text { for all } t \leq T-1 \\
\bar{\mu}^{t}=\mu^{t} & \text { for all } t \leq T-1 \\
\bar{I}^{T}=\emptyset &
\end{array}
$$

That is, $\left(\bar{\Gamma}^{t}, \bar{\mu}^{t}\right)_{t \leq T}$ is identical to $\left(\Gamma^{t}, \mu^{t}\right)_{t \leq T}$ up to period $T-1$, and all the students who would opt to play the spot mechanism in period $T$ in $\left(\Gamma^{t}, \mu^{t}\right)_{t \leq T}$ are now finalizing their match in period $T-1$ in $\left(\bar{\Gamma}^{t}, \bar{\mu}^{t}\right)_{t \leq T}$. So, $\bar{t}_{i}=T-1$ for each student $i \in I^{T}$. Since $\left(\Gamma^{t}, \mu^{t}\right)_{t \leq T}$ is gradually stable, so is $\left(\bar{\Gamma}^{t}, \bar{\mu}^{t}\right)_{t \leq T}$. Hence, $\bar{\mu}^{T-1}$ is a stable matching for $\bar{\Gamma}^{T-1}$, and since $\left(\bar{\Gamma}^{T-1}, \bar{\mu}^{T-1}\right)=\left(\Gamma^{T-1}, \mu^{T-1}\right)$, the matching $\mu^{T-1}$ is stable for $\Gamma^{T-1}$. Continuing this way until $t=1$ implies that $\mu^{t}$ is a stable matching for $\Gamma^{t}$, for $t=1, \ldots, T$. That is, $\varphi$ is a stable spot mechanism.

Let $\mathcal{M}_{F}^{\varphi}$ be gradually safe and $\varphi$ a stable spot mechanism. Let $\left(\Gamma^{t}, \mu^{t}\right)_{t \leq T}$ be the outcome of $\mathcal{M}_{F}^{\varphi}(\boldsymbol{\Gamma})$ for some $\boldsymbol{\Gamma}=\left(I^{1}, C,\left(P_{c}, q_{c}^{1}\right)_{c \in C}, \mathbf{P}_{I^{1}}\right)$. We show that the four conditions of Definition 8 are satisfied by $\left(\Gamma^{t}, \mu^{t}\right)_{t \leq T}$. Condition $(i)$ in Definition 8 is immediate since $\varphi$ is a stable spot mechanism and is thus individually rational. To show condition (ii), assume by way of contradiction that $\mu^{\bar{t}}(i) P_{i}^{t^{\prime}} \mu^{t^{\prime}}(i)$ for some $\bar{t}<t^{\prime}$. By Lemma $2, \mu^{\bar{t}}(i) P_{i}^{t} \mu^{t}(i)$ all $t \leq t^{\prime}$. But this contradicts $\mu^{\bar{t}+1} R_{i}^{\bar{t}+1} \mu^{\bar{t}}$, which holds since the mechanism is gradually safe. To show condition (iii), let $c \in C$ and $i \in I^{1}$ be such that $c P_{i}^{t^{\prime}} \mu^{t^{\prime}}(i)$. From Lemma $2, c P_{i}^{t} \mu^{t}(i)$ for all $t<t^{\prime}$. This implies that $\left|\mu^{t}(c)\right|=q_{c}^{t}$ for all $t \leq t^{\prime}$ since the spot mechanism satisfies non wastefulness at every period $t \leq t^{\prime}$. Finally, we show condition $(i v)$. Let $i, j \in I^{1}$ and $t$ such that $t_{j} \leq t \leq t_{i}$ and $c \in C$. such that $\mu^{t_{j}}(j)=c$ and $c P_{i}^{t} \mu^{t}(i)$ and suppose that $i P_{c} j$. By Lemma 2, it holds that $c P_{i}^{t_{j}} \mu^{t}(i)$, but this contradicts that the spot mechanism satisfies the no justified envy condition at period $t$.

\section{References}

Abdulkadiroğlu, A., Pathak, P. A., and Roth, A. E. (2005). The New York City high school match. American Economic Review, 95(2), 364-367.

Abdulkadiroğlu, A. and Sönmez, T. (2003). School Choice: A Mechanism Design Approach. American Economic Review, 93(3), 729-747. 
Akbarpour, M., Li, S., and Gharan, S. O. (2020). Thickness and Information in Dynamic Matching Markets. Journal of Political Economy, 128(3), 783-815.

Andersson, T., Dur, U., Ertemel, S., and Kesten, O. (2018). Sequential School Choice with Public and Private Schools. mimeo.

Antler, Y. (2015). Two-Sided Matching with Endogenous Preferences. American Economic Journal: Microeconomics, 7(3), 241-58.

Avery, C. and Levin, J. (2010). Early Admissions at Selective Colleges. American Economic Review, 100(5), 2125-56.

Baccara, M., Lee, S., and Yariv, L. (2020). Optimal Dynamic Matching. Theoretical Economics, 15, 1221-78.

Bloch, F. and Cantala, D. (2017). Dynamic Assignment of Objects to Queuing Agents. American Economic Journal: Microeconomics, 9(1), 88-122.

Bonkoungou, S. (2017). University Admissions Reform in France: An Analysis of a New Mechanism. mimeo.

Combe, J., Tercieux, O., and Terrier, C. (2018). The Design of Teacher Assignment: Theory and Evidence. mimeo.

Doğan, B. and Yenmez, B. (2018). When Does an Additional Stage Improve Welfare in Centralized Assignment? mimeo.

Doğan, B. and Yenmez, B. (2019). Unified versus Divided Enrollment in School Choice: Improving Student Welfare in Chicago. Games and Economic Behavior, 118, 366-73.

Doval, L. (2019). Dynamically Stable Matching. mimeo.

Dur, U. and Kesten, O. (2019). Sequential versus Simultaneous Assignment Systems and Two Applications. Economic Theory, 68, 251-83.

Echenique, F. and Pereyra, J. S. (2016). Strategic Complementarities and Unraveling in Matching Markets. Theoretical Economics, 11(1), 1-39.

Ekmekci, M. and Yenmez, B. (2019). Common Enrollment in School Choice. Theoretical Economics, 14, 1237-70. 
Ergin, H. I. (2002). Efficient Resource Allocation on the Basis of Priorities. Econometrica, 70(6), 2489-2497.

Gale, D. and Shapley, L. (1962). College Admissions and the Stability of Marriage. American Mathematical Monthly, 69(1), 9-15.

Halaburda, H. (2010). Unravelling in Two-Sided Matching Markets and Similarity of Preferences. Games and Economic Behavior, 69(2), 365-393.

Kadam, S. V. and Kotowski, M. H. (2018a). Multiperiod Matching. International Economic Review, 59(4), 1927-1947.

Kadam, S. V. and Kotowski, M. H. (2018b). Time Horizons, Lattice Structures, and Welfare in Multi-Period Matching Markets. Games and Economic Behavior, 112, 1 - 20.

Kagel, J. and Roth, A. (2000). The Dynamics of Reorganization in Matching Markets: A Laboratory Experiment Motivated by a Natural Experiment. The Quarterly Journal of Economics, 115(1), 201-235.

Kennes, J., Monte, D., and Tumennasan, N. (2014). The Day Care Assignment: A Dynamic Matching Problem. American Economic Journal: Microeconomics, 6(4), 362-406.

Kennes, J., Monte, D., and Tumennasan, N. (2019). Strategic Performance of Deferred Acceptance in Dynamic Matching Problems. American Economic Journal: Microeconomics, 11(2), 55-97.

Kesten, O. (2010). School Choice with Consent. The Quarterly Journal of Economics, 125(3), 1297-1348.

Kotowski, M. H. (2019). A Perfectly Robust Approach to Multiperiod Matching Problems. mimeo.

Manjunath, V. and Turhan, B. (2016). Two School Systems, One District: What to Do when a Unified Admissions Process is Impossible. Games and Economic Behavior, 95, 25-40.

Pereyra, J. S. (2013). A Dynamic School Choice Model. Games and Economic Behavior, $80,100-114$. 
Roth, A. E. (1984). The Evolution of the Labor Market for Medical Interns and Residents: A Case Study in Game Theory. Journal of Political Economy, 92(6), 991-1016.

Roth, A. E. (1985). The College Admissions Problem is not Equivalent to the Marriage Problem. Journal of Economic Theory, 36(2), 277-288.

Roth, A. E. (1986). On the Allocation of Residents to Rural Hospitals: a General Property of Two-Sided Matching Markets. Econometrica: Journal of the Econometric Society, pages $425-427$.

Roth, A. E. (1991). A Natural Experiment in the Organization of Entry-Level Labor Markets: Regional Markets for New Physicians and Surgeons in the United Kingdom. American Economic Review, 81(3), 415-40.

Segal, I. (2007). The Communication Requirements of Social Choice Rules and Supporting Budget Sets. Journal of Economic Theory, 136(1), 341-378.

Westkamp, A. (2012). An Analysis of the German University Admissions System. Economic Theory, 53(3), 561-589. 